\title{
RANDOM METHODS IN 3-MANIFOLD THEORY
}

\author{
ALEXANDER LUBOTZKY, JOSEPH MAHER AND CONAN WU
}

June 4, 2018

Contents

1. Introduction 1

1.1. Outline 2

1.2. Acknowledgments 3

2. Proof of the main theorem 4

3. Coarse geometry 10

4. Local estimates far from the disc set 17

5. Train tracks and shadows 21

6. Local estimates close to the disc set 25

7. Exponential decay for distance from the disc set 27

8. Exponential decay for Heegaard splitting distance 30

References 32

\section{INTRODUCTION}

Over the years, random methods have evolved into powerful techniques in several areas of mathematics. Most notably, as pioneered by Paul Erdős Erd59, the study of random graphs has become an important branch of contemporary graph theory. What is especially fascinating about this development is the fact that such techniques solved many problems which have nothing to do with probability: one can use random constructions to show the existence of a graph satisfying particular properties without constructing explicit examples. For example, graphs with both arbitrarily large girth and arbitrarily large chromatic number were shown to exist by random methods by Erdős Erd59] long before explicit examples were found by Lovász Lov68 and Lubotzky, Phillips and Sarnak LPS88.

The goal of this paper is to present similar methods within the world of 3manifolds. In 2006, Dunfield and W. Thurston DT06a presented a model of 'random' 3-manifolds by considering random walks on the mapping class group, and a theory of random 3-manifolds is starting to emerge (DT06b, Riv08, Kow08, Mah10, DW11, Mah11). Here we will use this theory to prove the following existence result, which a priori has nothing to do with randomness. 
Theorem 1. For any integers $k$ and $g$ with $g \geq 2$, there exist infinitely many closed hyperbolic 3-manifolds which are integral homology 3-spheres with Casson invariant $k$ and Heegaard genus equal to $g$.

In fact, results announced by Brock and Souto BS09 would show that the volume of these 3 -manifolds tends to infinity.

There is however a difference between our methodology and the common practice of random methods in graph theory. In graph theory, usually one proves " $0-1$ laws" and existence is shown by proving that "most" objects satisfy the desired property, even though no explicit examples are given. Here our considerations will be somewhat more delicate: we will have to compare rates of decay of various properties along random walks, and the difference between them will ensure the existence of the desired manifolds. We hope that our results will be an initial example of applying random methods to 3-manifolds.

1.1. Outline. In this section we give a brief outline of the proof. By a classical result (see, for example Hem76]), every closed 3-manifold can be obtained by gluing two genus $g$ handlebodies along their boundary surfaces $S_{g}$, and the minimum such $g$ is called the Heegaard genus of the manifold. As isotopic gluing maps give homeomorphic 3-manifold, every element $\phi$ of the mapping class group $\operatorname{MCG}\left(S_{g}\right)$ will give a 3-manifold $M(\phi)$ of Heegaard genus at most $g$. The main idea of DT06a is that a random walk on $\mathrm{MCG}\left(S_{g}\right)$ gives rise to a random model of 3-manifolds with Heegaard genus at most $g$; one may also consider random walks on any subgroup $H$ of $\operatorname{MCG}\left(S_{g}\right)$.

If $w_{n}$ is a random walk on a group $H$ generated by the probability distribution $\mu$, and $Y$ is a subset of $H$, we say that $Y$ is exponentially small with respect to $\mu$ if the probability of visiting $Y$ decays exponentially fast with $n$. We say the set $Y$ is exponentially large with respect to $\mu$ if the complement of $Y$ is exponentially small. We will often just write exponentially small or exponentially large if the probability distribution $\mu$ is clear from context. We do not necessarily require that $\mu$ be symmetric, however, we always require that the semi-group generated by the support of $\mu$ is a group. The main idea of the proof is to find a specifically chosen finitely generated subgroup $H$ of the Torelli subgroup $\mathcal{T}$ of the $\operatorname{MCG}\left(S_{g}\right)$, and for random walks on this subgroup we show:

(a) The set of elements of $H$ giving rise to hyperbolic manifolds is exponentially large.

(b) The set elements of $H$ giving rise to manifolds of Heegaard genus exactly $g$ is exponentially large.

(c) The Casson invariant restricted to $H$ is a homomorphism from $H$ onto $\mathbb{Z}$.

Theorem 1 follows from (a), (b) and (c). Indeed by standard results from random walks on $\mathbb{Z}$, the random walk $w_{n}$ visits each $k \in \mathbb{Z}$ with probability $1 / \sqrt{n}$, for $n$ 
sufficiently large. By (a) and (b) most of these visits will give rise to hyperbolic manifolds of Heegaard genus exactly $g$. The resulting manifolds will also be integral homology spheres, as we shall choose the subgroup $H$ to be contained in the Torelli subgroup.

In more detail, we choose $H$ to be a subgroup of $\mathcal{K}$, the group generated by Dehn twists in separating curves. By a result of Morita [Mor97], the Casson invariant $\lambda: \mathcal{K} \rightarrow \mathbb{Z}$ is an epimorphism. While $\mathcal{K}$ is not expected to be finitely generated, we choose a sufficiently large finitely generated subgroup $H$ of $\mathcal{K}$ for which $\lambda$ restricted to $H$ still has surjective image in $\mathbb{Z}$.

For this subgroup $H$ we prove properties (a) and (b). Moreover, much of what we prove holds for complete subgroups of the mapping class group, i.e. subgroups whose limit set is equal to the full boundary. To put this in perspective, let us mention that Maher Mah10 showed that in complete, finitely generated subgroups, the probability that a random walk gives rise to a manifold which is hyperbolic, and of Heegaard genus $g$, tends to 1, but without the exponential decay estimate. We now have the following result which is of independent interest. We shall write $\operatorname{supp}(\mu)$ for the support of $\mu$, i.e. all group elements $g \in G$ with $\mu(g)>0$. We shall write $\langle\operatorname{supp}(\mu)\rangle_{+}$for the semi-group generated by $\operatorname{supp}(\mu)$, and we shall refer to this as the semi-group support of $\mu$.

Theorem 2. Let $L$ be a finitely generated complete subgroup of $M C G\left(S_{g}\right)$, then for any finitely supported probability distribution $\mu$, whose semi-group support $\langle\operatorname{supp}(\mu)\rangle_{+}$ is equal to $L$, the set of elements which yield hyperbolic manifolds of Heegaard genus equal to $g$ is exponentially large.

This result is new even for $L=\operatorname{MCG}\left(S_{g}\right)$ or the Torelli group. In fact, we prove a more general result, which includes the subgroup $H$ as before, which need not be complete.

The real work in proving Theorem 2 is to control the Heegaard splitting distance. It is known that if the Heegaard distance of $\phi \in \operatorname{MCG}\left(S_{g}\right)$ is at least 3 then $M(\phi)$ is hyperbolic, by work of Kobayashi Kob88a, Hempel [Hem01] and Perelman MT07, and if the spitting distance is at least $2 g+1$ then $M(\phi)$ has Heegaard genus exactly $g$, by work of Scharlemann and Tomova ST06. In fact, we show that the Heegaard splitting distance of the random 3-manifold grows linearly with exponential decay. Results announced by Brock and Souto BS09] would then imply that the manifolds obtained in Theorem 1 have arbitrarily large volume.

1.2. Acknowledgments. The authors are grateful to Martin Bridson, Nathan Dunfield, Benson Farb, Alexander Holroyd, Justin Malestein and Yair Minksy for useful discussions. We acknowledge support by ERC, NSF and ISF. The second 
author was supported by PSC-CUNY award TRADB-45-17 and Simons Foundation grant CGM 234477. The third author thanks GARE network and the warm hospitality of Hebrew University.

\section{Proof of THE MAIN THEOREM}

Before starting the proof we give some background and fix notation. Let $S_{g}$ be a closed orientable surface of genus $g$. We shall write $\operatorname{MCG}\left(S_{g}\right)$ for the mapping class group of $S_{g}$, which is the group of all orientation preserving homeomorphisms of $S_{g}$ up to isotopy. We shall write $\mathcal{C}\left(S_{g}\right)$ for the curve complex of $S_{g}$, which is a simplicial complex, whose vertices are given by isotopy classes of simple closed curves, and whose simplices are spanned by collections of disjoint simple closed curves. A handlebody $U$ is a compact 3-manifold with boundary, homeomorphic to a regular neighbourhood of an embedded graph in $\mathbb{R}^{3}$, and handlebodies are classified up to homeomorphism by the genus of their boundary surfaces. Given an identification of the surface $S_{g}$ with the boundary of a genus $g$ handlebody, the handlebody group is the subgroup of the mapping class group consisting of those mapping class group elements which extend over the handlebody, i.e. they arise as restrictions of self-homeomorphisms of the handlebody. The disc set $\mathcal{D}$ is defined to be the subset of the curve complex $\mathcal{C}\left(S_{g}\right)$ consisting of all simple closed curves in $S_{g}$ which bound discs in the handlebody. A genus $g$ Heegaard splitting of a closed orientable 3-manifold $M$ is an embedding of $S_{g}$ in $M$ which divides $M$ into two handlebodies $U$ and $U^{\prime}$, and we shall denote their corresponding discs sets by $\mathcal{D}$ and $\mathcal{D}^{\prime}$ respectively. Any two handlebodies of the same genus are homeomorphic, so for any pair of discs sets $\mathcal{D}$ and $\mathcal{D}^{\prime}$, corresponding to two identifications of $S_{g}$ with the boundaries of the handlebodies, there is a mapping class group element $h$ such that $\mathcal{D}^{\prime}=h \mathcal{D}$. The mapping class group element is not unique, but any two choices differ by composition with elements of the handlebody group.

In particular, a Heegaard splitting of $S^{3}$ is an embedded copy of the surface $S_{g}$ in the standard 3-sphere, separating the sphere into two genus $g$ handlebodies. In fact, for $S^{3}$, such a splitting is unique up to isotopy, and from now on we shall fix a pair of discs sets $\mathcal{D}$ and $\mathcal{D}^{\prime}$, and a mapping class group element $h_{S^{3}}$ with $h_{S^{3}} \mathcal{D}=\mathcal{D}^{\prime}$, arising from a genus $g$ Heegaard splitting of $S^{3}$. Given an element $\phi$ of the mapping class group, we may consider the Heegaard splitting obtained by composing the gluing map $h_{S^{3}}$ with $\phi$, i.e. the Heegaard splitting with disc sets $\mathcal{D}$ and $\phi h_{S^{3}} \mathcal{D}$, and we shall just write $M(\phi)$ for the resulting 3-manifold. The 3-manifold $M(\phi)$ is an integral homology sphere if and only if $\phi$ lies in the Torelli subgroup $\mathcal{T}$ of the mapping class group $\operatorname{MCG}\left(S_{g}\right)$, i.e. the subgroup which acts trivially on the homology of the surface.

We will often consider the orbit map from the mapping class group $\operatorname{MCG}\left(S_{g}\right)$ to the curve complex $\mathcal{C}(S)$, which sends $\phi \mapsto \phi x_{0}$, for some choice of basepoint $x_{0}$. 
Our particular choice of disc sets $\mathcal{D}$ and $\mathcal{D}^{\prime}$ intersect, i.e. in the unique genus $g$ Heegaard splitting of $S^{3}$ there is a curve on the Heegaard surface which bounds a disc on both sides, and it will be convenient for us to choose a basepoint $x_{0}$ which lies in both $\mathcal{D}$ and $\mathcal{D}^{\prime}$. We remark that this is for convenience only, as the argument works for any other choice of basepoint, possibly with slightly different constants. Furthermore, the argument works for any other choice of initial disc sets, $\mathcal{D}$ and $\mathcal{D}^{\prime}$, again possibly with different constants, as long as the Heegaard splitting corresponding to the two disc sets is an integer homology sphere; this is equivalent to starting the random walk at some other element of the mapping class group, instead of the usual choice of the identity element.

Let $\mu$ be a probability distribution on $\operatorname{MCG}\left(S_{g}\right)$ with finite support. The random walk on $\operatorname{MCG}\left(S_{g}\right)$ generated by $\mu$ is the Markov chain with the transition probability from $x$ to $y$, denoted $p(x, y)$, equal to $\mu\left(x^{-1} y\right)$. We will always assume that we start at the identity at time zero, and we will write $w_{n}$ for the location of the random walk at time $n$. The probability distribution $\mu$ need not be symmetric, but we shall always assume that the semi-group generated by the support of $\mu$ is a subgroup of the mapping class group. Taking the probability of the random walk landing in a particular set gives rise to a way of measuring the size of subsets of our group: we say that a subset $E \subseteq G$ is exponentially small if there exists numbers $K, c<1$ such that for every $n \in \mathbb{N}$,

$$
\mathbb{P}\left(w_{n} \in E\right) \leq K c^{n}
$$

We will call a subset of $G$ exponentially large if its complement is exponentially small.

Given $g \in \operatorname{MCG}\left(S_{g}\right)$ which is pseudo-Anosov, let $\mathcal{L}^{s}(g)$ and $\mathcal{L}^{u}(g)$ denote the stable and unstable laminations of $g$. A finitely generated subgroup $G$ of $\operatorname{MCG}\left(S_{g}\right)$ is said to be sufficiently large if it contains two pseudo-Anosov elements $\varphi, \psi$ with distinct stable and unstable laminations, namely

$$
\left\{\mathcal{L}^{s}(\varphi), \mathcal{L}^{u}(\varphi)\right\} \cap\left\{\mathcal{L}^{s}(\psi), \mathcal{L}^{u}(\psi)\right\}=\phi
$$

The subgroup $G$ is complete if the endpoints of its pseudo-Anosov elements are dense in $\mathcal{P} \mathcal{M L}$, Thurston's boundary for Teichmüller space. Ivanov Iva92 showed that an infinite normal subgroup contains a pseudo-Anosov element, and the orbit under $\operatorname{MCG}\left(S_{g}\right)$ of any point in $\mathcal{P} \mathcal{M L}$ is dense, so every infinite normal subgroup of $\operatorname{MCG}\left(S_{g}\right)$ is complete and sufficiently large.

Let $\mathcal{K}$ be the subgroup of $\mathcal{T}$ generated by Dehn twists along separating curves. As $\mathcal{K}$ is a normal subgroup, it is complete. In fact, as discussed in Farb and Margalit FM12, Chapter 6], the group $\mathcal{K}$ coincides with the second Torelli group, $\mathcal{T}^{2}\left(S_{g}\right)$, also known as the Johnson kernel, defined as the kernel of the action of $\mathcal{T}\left(S_{g}\right)$ on the quotient $\Gamma /\left[\Gamma, \Gamma^{\prime}\right]$ where $\Gamma=\pi_{1}\left(S_{g}\right)$ and $\Gamma^{\prime}=[\Gamma, \Gamma]$ is the commutator subgroup. 
The Casson invariant $\lambda$ of closed orientable integral homology spheres takes values in $\mathbb{Z}$. Casson defined the invariant in terms of $S U(2)$ representations arising from a Heegaard splitting of the manifold, see for example Akbulut and McCarthy AM90. The Casson invariant of $S^{3}$ is equal to 0 and the Casson invariant of the Poincaré homology sphere is equal to 1 . As the sign of $\lambda(M)$ changes if you reverse the orientation on $M$, and the Casson invariant is additive under connect sum, taking connect sums of Poincaré homology spheres with appropriate orientation gives examples of manifolds with any integral value for their Casson invariant, though these manifolds will not be hyperbolic, and their Heegaard genera are unbounded.

We shall use the following property of the Casson invariant which is due to Morita [Mor97].

Theorem 3. (Morita Mor97]) The Casson invariant $\lambda: \mathcal{K} \rightarrow \mathbb{Z}$ is a homomorphism.

In fact, this homorphism is surjective, and we show this by constructing an explicit element of the Johnson kernel $\mathcal{K}$ which maps to a generator of $\mathbb{Z}$.

Lemma 4. The Casson invariant homomorphism $\lambda: \mathcal{K} \rightarrow \mathbb{Z}$ is surjective.

Given a knot $\kappa$ in $S^{3}$, we will write $S^{3}+(p / q) \kappa$ for the 3 -manifold obtained by $(p / q)$-Dehn surgery along $\kappa$ in $S^{3}$.

Proof. By the surgery formula for the Casson invariant, see for example Sav02, Section 3.2.8], for Dehn surgeries on the trefoil knot $\kappa$ we have

$$
\left|\lambda\left(S^{3}+\frac{1}{m+1} \kappa\right)-\lambda\left(S^{3}+\frac{1}{m} \kappa\right)\right|=1,
$$

independent of the integer $m$.

Now we can embed $\kappa$ as a separating curve $C$ in the closed genus two surface as follows:
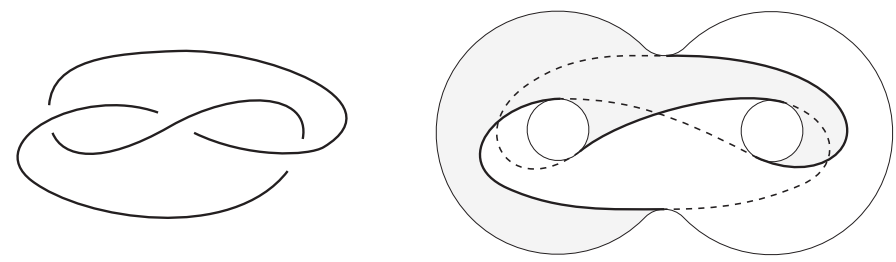

Figure 1. Embedded separating trefoil

It is a standard fact (see, for example, Sav02, Section 3.2.5]) that the $S^{3}+\frac{1}{m} \kappa$ is the same as taking the standard embedding of $S_{2}$ in $S^{3}$ and gluing the two handlebodies with $m$ Dehn twists along the embedded copy of $\kappa$.

Let $\tau \in \mathcal{K}$ be the Dehn twist along $C$, so we have

$$
\left|\lambda\left(\tau^{2}\right)-\lambda(\tau)\right|=1
$$


This finishes the proof when $g=2$.

As the Casson invariant is additive under connected sums, hence for any $g>2$ we may simply add handles on the standard embedding of $S_{2}$ away from the embedding of $\kappa$ and obtain the same manifold by applying $m$ Dehn twists along $\kappa$.

Since $\mathcal{K}$ is the group generated by Dehn twists along separating curves in $S_{g}$, we deduce that for all $m, S^{3}+\frac{1}{m} \kappa$ are manifolds obtained as $M(\phi)$ for some $\phi \in \mathcal{K}$. We conclude there are always consecutive integers in the image $\lambda(\mathcal{K})$, i.e. $\left.\lambda\right|_{\mathcal{K}}$ is surjective.

Given subsets $A$ and $B$ of the curve complex, we will write $d(A, B)$ for the minimum distance $d(a, b)$ between elements $a \in A$ and $b \in B$. The Heegaard splitting distance of a Heegaard splitting, as defined by Kobayashi Kob88b and Hempel Hem01, is the distance between the two disc sets determined by the handlebodies of the splittings. In our notation, given a mapping class group element $\phi$, the corresponding 3-manifold $M(\phi)$ has Heegaard splitting distance

$$
d_{\mathrm{sp}}(M(\phi))=d\left(\mathcal{D}, \phi h_{S^{3}} \mathcal{D}\right) .
$$

In Mah10, it was shown that for random walks on finitely generated complete subgroups of the mapping class group, the splitting distance $d_{\mathrm{sp}}\left(M\left(w_{n}\right)\right)$ grows linearly with $n$, i.e. there is a number $L>0$ such that $P\left(d_{\mathrm{sp}}\left(M\left(w_{n}\right)\right) \geqslant L n\right) \rightarrow 1$ as $n \rightarrow \infty$. Although $\mathcal{K}$ is complete, it is not expected to be finitely generated, so we need a stronger version of this result which works for subgroups, which need not be complete, and which furthermore shows that the probability tends to 1 exponentially fast. We say a sequence of random variables $\left\{X_{n}\right\}_{n \in \mathbb{N}}$ grow linearly with exponential decay if there are numbers $K, L>0$ and $c<1$ such that

$$
\mathbb{P}\left(X_{n} \leqslant L n\right) \leqslant K c^{n},
$$

for all $n$.

Theorem 5. For any complete subgroup $G$ of $M C G\left(S_{g}\right)$, there is a finitely generated subgroup $H<G$, such that for any finitely supported probability distribution $\mu$, whose semi-group support $\langle\operatorname{supp}(\mu)\rangle_{+}$is a subgroup containing $H$, the Heegaard splitting distance $d_{s p}\left(M\left(w_{n}\right)\right)$, of a random walk of length $n$ generated by $\mu$, grows linearly with exponential decay, i.e. there are numbers $K, L>0$ and $c<1$ such that

$$
\mathbb{P}\left(d_{s p}\left(M\left(w_{n}\right)\right) \leqslant L n\right) \leqslant K c^{n} .
$$

Any sufficiently large normal subgroup of the mapping class group is complete, so in the result above $G$ may be taken to be the entire $\operatorname{MCG}\left(S_{g}\right)$, the Torelli group, or the Johnson kernel $\mathcal{K}$. If $G$ is finitely generated, we may choose the support of $\mu$ to generate $G$. The mapping class group $\operatorname{MCG}\left(S_{g}\right)$, is finitely generated, as shown by Dehn Deh38 and Lickorish Lic64], as is the Torelli group, for $g>2$, as shown by Johnson Joh83. For $g=2$, the Torelli group is not finitely generated, as shown 
by McCullough and Miller MM86, and it is not currently known whether or not $\mathcal{K}$ is finitely generated.

We postpone the proofs of Theorem 5 to the later sections.

Kobayashi Kob88a and Hempel [Hem01] showed that if the splitting distance is greater than 2 then the 3-manifold is irreducible, atoroidal and not Seifert-fibered, and so is hyperbolic by Perelman's proof of Thurston's geometrization conjecture MT07. Scharleman and Tomova ST06] showed that if the Heegaard splitting distance $d_{\mathrm{sp}}(M(h))$ is greater than $2 g$, then the Heegaard genus of the resulting 3 -manifold is equal to $g$. As the set $H_{2 g}$ of elements in $H$ that induce 3-manifolds of splitting distance at most $2 g$ is exponentially small in $H$, this implies that its complement, the set $\left(H_{2 g}\right)^{c}$, all of whose elements correspond to 3-manifolds which are hyperbolic and have Heegaard genus equal to $g$, is exponentially large in $H$.

Now we are ready to put the parts together and obtain the main theorem.

Proof. (of Theorem 11) Consider $\mathcal{K}$, which is a complete subgroup of $\operatorname{MCG}\left(S_{g}\right)$. By Theorem 5, there is a finitely generated subgroup $H_{0}<\operatorname{MCG}\left(S_{g}\right)$ such that for any finitely supported probability distribution $\mu$ whose support generates a subgroup $H$ containing $H_{0}$, the splitting distance $d_{\mathrm{sp}}\left(M\left(w_{n}\right)\right)$ grows linearly with exponential decay.

We shall consider the subgroup $H$ generated by $\left\{H_{0} \cup \tau\right\}$, where $\tau \in \mathcal{K}$ is the element previously constructed in Lemma 4 for which $\lambda(\tau)=1$. This ensures that the homomorphism $\lambda: H \rightarrow \mathbb{Z}$ is surjective. The subgroup $H$ is finitely generated, as $H_{0}$ is finitely generated. We may now choose a symmetric random walk, supported on a finite generating set for $H$, and the image of this random walk under the homorphism $\lambda$ is a symmetric finite range random walk on $\mathbb{Z}$. Furthermore, we may assume that the image of the random walk on $\mathbb{Z}$ is irreducible, for example by adding an element to the generating set which maps to 0 .

We shall write $H_{2 g}$ to denote the subset of $H$ consisting of group elements which give rise to Heegaard splittings of distance less than or equal to $2 g$. Suppose some $k \in \mathbb{Z}$ is not achieved as the Casson invariant of a hyperbolic homology sphere with Heegaard genus $g$, then in particular we have

$$
\lambda^{-1}(k) \subseteq H_{2 g} .
$$

The set on the right hand side is exponentially small by Theorem 5 .

Now since $\lambda$ is a homomorphism on $H$, it projects the random walk on $H$ to an irreducible Markov process on $\mathbb{Z}$, and for a symmetric finite range random walk, at step $n$,

$$
\mathbb{P}\left(\lambda\left(w_{n}\right)=k\right) \geqslant c / \sqrt{n}
$$

with some small number $c$, see for example Lawler and Limic LL10, Section 2.1]. This contradicts the assumption of $\lambda^{-1}(k)$ being exponentially small. 
Let us now sketch the proof of Theorems 5 and 2. We now give a brief overview of the argument showing that the Heegaard splitting distance $d_{\mathrm{sp}}\left(M\left(w_{n}\right)\right)$ grows linearly with exponential decay.

Let $x_{0}$ be a basepoint in the complex of curves $\mathcal{C}\left(S_{g}\right)$, and consider the image of the random walk under the orbit map $w_{n} \mapsto w_{n} x_{0}$. A key intermediate result is to show that for a random walk on a suitably chosen subgroup $H$ of $\mathcal{K}$, the distance of the sample path from the disc set, $d\left(\mathcal{D}, w_{n} x_{0}\right)$, grows linearly in $n$ with exponential decay, and we now give an outline of the argument for this result, omitting certain technical details.

We shall write $\mathbb{N}_{0}$ to denote the set of non-negative integers. Consider the sequence of random variables $X_{n}=d\left(\mathcal{D}, w_{n} x_{0}\right)$ with values in $\mathbb{N}_{0}$. This is not a Markov chain, but the conditional probabilities

$$
\mathbb{P}\left(X_{n+1}=j \mid w_{n}=\phi\right)
$$

are well-defined. We shall show the following pair of "local" estimates for these conditional probabilities. We state approximate versions of the properties here, and precise versions later on.

(1) If the sample path location $w_{n} x_{0}$ is reasonably far from the disc set, then the probability that after $m$ steps you have moved a distance $r$ closer to the disc set decays exponentially in $r$, i.e. there is some $q<1$ such that

$$
\mathbb{P}\left(X_{n+m}=t-r \mid w_{n}=\phi \text { with } d\left(\mathcal{D}, \phi x_{0}\right)=t\right) \leqslant q^{r+1} .
$$

(2) If the sample path location $w_{n} x_{0}$ is close to the disc set, then there is a definite chance $\varepsilon>0$ that after $m$ steps you have moved a reasonably distance $r$ away from the disc set, i.e.

$$
\mathbb{P}\left(X_{n+m} \geqslant r \mid w_{n}=\phi \text { with } d\left(\mathcal{D}, \phi x_{0}\right)=0\right) \geqslant \varepsilon .
$$

We briefly indicate some of the details that need to be addressed. We need to obtain the estimates above for all $n$ and some fixed but suitably large $m$. It is also more convenient to work with a coarse version of distance, in which we choose $X_{n}$ to be the integer part of $d\left(\mathcal{D}, w_{n} x_{0}\right) / R$, for some reasonably large $R$, rather than $d\left(\mathcal{D}, w_{n} x_{0}\right)$ itself.

In Section 3 we review some basic results in coarse geometry, before using these to show the first property in Section 4 . Then in Section 5 we review some basic results about train tracks and shadow sets, and then use these to show the second property in Section 6 .

In Section 7 we show how to use the two properties above to show that $d\left(\mathcal{D}, w_{n} x_{0}\right)$ grows linearly, with exponential decay, and we now describe our approach.

The sequence of random variables $\left\{X_{n}\right\}$ does not arise from a Markov chain, but we can compare the distributions of the $\left\{X_{n}\right\}$ with the distributions $\left\{Y_{n}\right\}$ arising from a Markov chain on $\mathbb{N}_{0}$, which never increases by more than one unit per step, 
and has transition probabilities given by $p(0,0)=1-\varepsilon$, and $p(t, t-r)=q^{r+1}$, for $r \geqslant 0$. The first few vertices of this Markov chain are illustrated in Figure 2 .

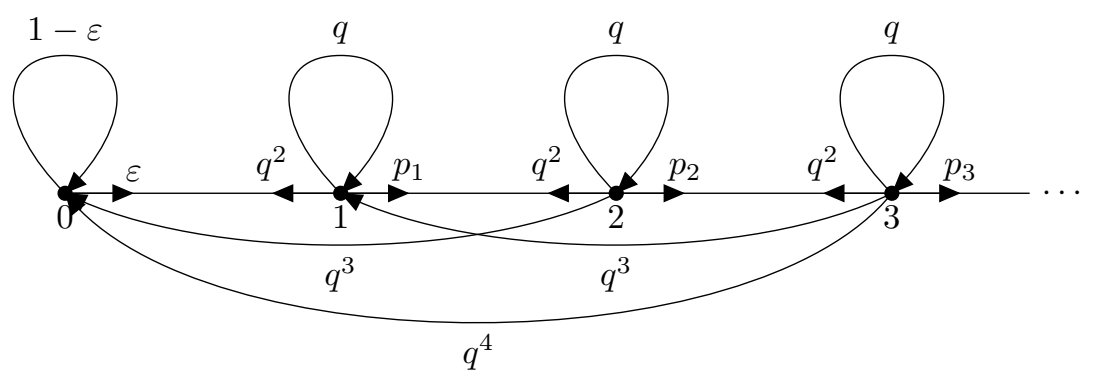

Figure 2. The Markov chain $\left(\mathbb{N}_{0}, P\right)$, where $p_{i}=1-q-q^{2}-\cdots-q^{i+1}$.

Intuitively, it is always more likely that the random variables $\left\{X_{n}\right\}$ move further to the right than the random variables $\left\{Y_{n}\right\}$ arising from the Markov chain. This means that the distribution of each $Y_{n}$ will have greater weight on small values than the distribution for the corresponding $X_{n}$, or more precisely $F_{X_{n}}(t) \leqslant F_{Y_{n}}(t)$ for all $t$, where $F_{X_{n}}$ and $F_{Y_{n}}$ are the cumulative probability functions for $X_{n}$ and $Y_{n}$ respectively. This property is usually described by saying that the random variables $X_{n}$ stochastically dominate the random variables $Y_{n}$, written as $Y_{n} \prec X_{n}$. A standard argument from the theory of Markov chains shows that the random variables $Y_{n}$ arising from the Markov chain satisfies the linear progress with exponential decay property that we require, and so this implies the linear progress with exponential decay property for the $X_{n}$.

Finally, in Section 8 we use some more coarse geometry to extend this to show that $d\left(\mathcal{D}, w_{n} h_{S^{3}} \mathcal{D}\right)$ grows linearly with exponential decay.

\section{CoARse GeOMetry}

Let $(X, d)$ be a $\delta$-hyperbolic space, which need not be locally compact. Recall that the Gromov product of two points $y$ and $z$ in $X$, with respect to a basepoint $x \in X$, is defined to be

$$
(y \cdot z)_{x}=\frac{1}{2}(d(x, y)+d(x, z)-d(y, z)) .
$$

This is equal to the distance from $x$ to a geodesic from $y$ to $z$ up to bounded additive error. Given a basepoint $x \in X$, and a number $R \geqslant 0$, we define the shadow set of a point $y \in X$, with parameter $R$, to be

$$
S_{x}(y, R)=\left\{z \in X \mid(y \cdot z)_{x} \geqslant d(x, y)-R\right\}
$$

We start with an elementary observation concerning distances in $\delta$-hyperbolic spaces. Suppose you travel from a point $x$ to a point $y$ along a geodesic $\gamma$, and 
then from the point $y$ to $z$ along a geodesic $\gamma^{\prime}$. By thin triangles, the two geodesics $\gamma$ and $\gamma^{\prime}$ fellow travel for some distance near $y$, before moving apart, and we can think of the length of the fellow-travelling segments as measuring the overlap of the two geodesics.

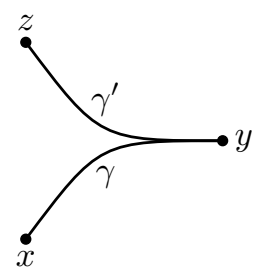

FiguRE 3. Overlapping geodesics

The total distance from $d(x, z)$ is equal to $d(x, y)+d(y, z)$ minus approximately twice this overlap. It is convenient to use the Gromov product $(x \cdot z)_{y}$ as a measure of this overlap, and then it follows immediately from the definition of the Gromov product that

$$
d(x, z)=d(x, y)+d(y, z)-2(x \cdot z)_{y},
$$

i.e. we can estimate the distance from $x$ to $z$ as the sum of the distances from $x$ to $y$ and $y$ to $z$, together with a term involving the Gromov product.

The aim of this section is to show similar estimates for the case in which the point $x$ is replaced by a quasiconvex set, and also for the case in which both the points $x$ and $z$ are replaced with quasiconvex sets, as illustrated in Figure 4 , where $x$ is the closest point on $D$ to $y$ and $z$ is the closest point on $E$ to $y$.
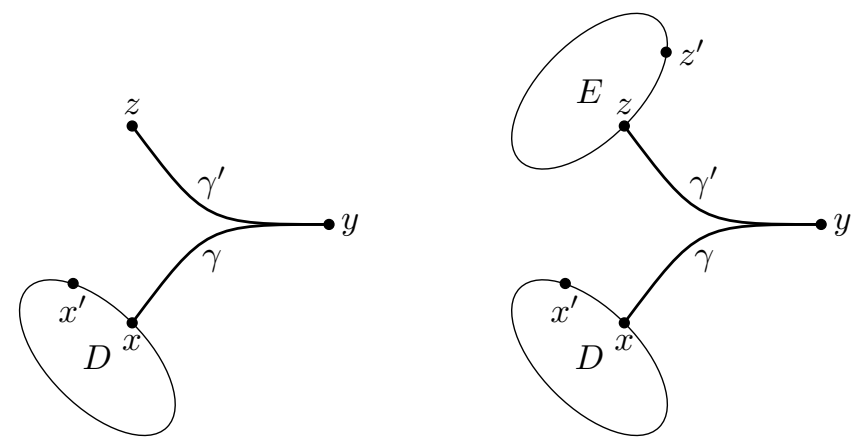

FIGURE 4. Estimating distances between quasiconvex sets and points.

Recall that a set $D \subset X$ is $Q$-quasiconvex if any geodesic connecting two points of $D$ is contained in a $Q$-neighbourhood of $D$. We now state the two results we will use in the later sections. In the first result, we replace $x$ with a quasiconvex set 
$D$, and find a lower bound on $d(D, z)$, using an additional hypothesis on $(x \cdot z)_{y}$. Furthermore, we show that the Gromov product based at $y$ of $z$ with any point in $D$ is equal to $(x \cdot z)_{y}$ up to bounded additive error depending only on $\delta$ and $Q$.

Proposition 6. Let $(X, d)$ be a $\delta$-hyperbolic space, which need not be locally compact. Given a number $Q$, there are numbers $A, B$ and $C$, which only depend on $\delta$ and $Q$, such that if $D$ is a $Q$-quasiconvex set, and $x$ is a closest point in $D$ to $y$, then for any point $z$ with

$$
(x \cdot z)_{y} \leqslant d(x, y)-A,
$$

then

$$
d(D, z) \geqslant d(x, y)+d(y, z)-2(x \cdot z)_{y}-B .
$$

Furthermore, for any point $x^{\prime} \in D$,

$$
\left|(x \cdot z)_{y}-\left(x^{\prime} \cdot z\right)_{y}\right| \leqslant C .
$$

For the second result, we produce a similar estimate with both $x$ and $z$ replaced with quasiconvex sets $D$ and $E$, with additional hypotheses on $(x \cdot z)_{y}$. Furthermore, we show that the Gromov product based at $y$ of any point in $D$ with any point in $E$, is equal to $(x \cdot z)_{y}$, again up to bounded additive error depending only on $\delta$ and $Q$.

Proposition 7. Let $(X, d)$ be a $\delta$-hyperbolic space, which need not be locally compact. Given a number $Q$, there are numbers $A, B$ and $C$, which only depend on $\delta$ and $Q$, such that if $D$ and $E$ are $Q$-quasiconvex sets, and $x$ is a closest point in $D$ to $y$, and $z$ is a closest point in $E$ to $y$, then if

$$
(x \cdot z)_{y} \leqslant \min \{d(x, y), d(y, z)\}-A,
$$

then

$$
d(D, E) \geqslant d(x, y)+d(y, z)-2(x \cdot z)_{y}-B .
$$

Furthermore, for any points $x^{\prime} \in D$ and $z^{\prime} \in E$,

$$
\left|(x \cdot z)_{y}-\left(x^{\prime} \cdot z^{\prime}\right)_{y}\right| \leqslant C .
$$

These results follow from standard arguments in coarse geometry, but we give full details for the convenience of the reader. We recall the following result regarding approximate trees in $\delta$-hyperbolic spaces, see for example Ghys and de la Harpe GdlH90, Section 2.2].

Lemma 8 (Approximate tree). Let $(X, d)$ be a $\delta$-hyperbolic space, which need not be locally compact. Then there is a number $K_{T}$, which depends only on $\delta$, such that for any finite collection of points $x_{1}, \ldots, x_{n}$, there is a geodesic tree $T$ in $X$ containing the $x_{i}$, such that

$$
d_{T}(x, y)-K_{T} n \leqslant d(x, y) \leqslant d_{T}(x, y)
$$

for all points $x$ and $y$ in $T$. We call $T$ the approximate tree determined by the $x_{i}$. 
As the Gromov product is defined in terms of distances, if we write $(y \cdot z)_{x}^{T}$ for the Gromov product in the tree $T$ with vertices $\left\{x_{1}, \ldots x_{n}\right\}$, then $\left|(y \cdot z)_{x}^{T}-(y \cdot z)_{x}\right|$ is bounded by a number, in fact $3 K_{T} n / 2$, which only depends on $\delta$ and $n$, where $n$ is the number of vertices in the tree.

Recall that any three distinct points in a tree determine a unique center, namely the unique point that lies in the intersection of the three geodesics connecting the three possible pairs of points. It follows from the definition of the Gromov product that for any points $x, y$ and $z$ in a tree $T$, if $v$ is the center for $x, y$ and $z$, then $(x \cdot y)_{z}^{T}=d_{T}(z, v)$.

Let $I$ be a connected subinterval of $\mathbb{R}$. We say a path $\gamma: I \rightarrow X$ is a $(K, c)$ quasigeodesic if

$$
\frac{1}{K}|x-y|-c \leqslant d(\gamma(x), \gamma(y)) \leqslant K|x-y|+c,
$$

for all $x$ and $y$ in $\mathbb{R}$. In a $\delta$-hyperbolic space, quasigeodesics are contained in bounded neighbourhoods of geodesics. This is often known as the Morse Lemma, see for example Bridson and Haefligger BH99, Theorem III.H.1.7].

Lemma 9. Let $\gamma$ be a $(K, c)$-quasigeodesic in a $\delta$-hyperbolic space. Then there is a number $L$, depending only on $K, c$ and $\delta$, such that $\gamma$ is contained in an $L$ neighbourhood of the geodesic connecting its endpoints.

As geodesics in the approximate tree $T$ are $\left(1, K_{T} n\right)$-quasigeodesics, this implies that any geodesic in $T$ is contained in an $L_{n}$-neighbourhood of the geodesic in $X$ connecting its endpoints, for some number $L_{n}$, depending only on $\delta$ and $n$.

Proposition 10. Let $(X, d)$ be a $\delta$-hyperbolic space, which need not be locally compact. Let $D$ be a $Q$-quasiconvex set, and let $y$ be a point in $X$. Let $x$ be the closest point in $D$ to $y$, and let $x^{\prime}$ be an arbitrary point in $D$. Let $T$ be an approximate tree determined by a set of $n$ points, which include $x, x^{\prime}$ and $y$, and let $v$ be the center of $x, x^{\prime}$ and $y$ in $T$. Then there is a number $A$, which only depends on $\delta, Q$ and $n$, such that

$$
d(x, v) \leqslant A
$$

Although the constant $A$ depends on the number of points determining the approximate tree $T$, we will only consider approximate trees determined by at most 5 points.

Proof. (of Proposition 10,) Let $D$ be a $Q$-quasiconvex set, let $y$ be a point in $X$, let $x$ be the closest point in $D$ to $y$, and let $x^{\prime}$ be any other point in $D$. Let $T$ be an approximate tree containing $n$ points, which include $x, x^{\prime}$ and $y$, and let $v$ be the center of $x, x^{\prime}$ and $y$ in $T$. In particular, the minimal subtree in $T$ containing $x, x^{\prime}$ and $y$ also contains $v$, and has the configuration illustrated in Figure 5 possibly with edges of zero length. 


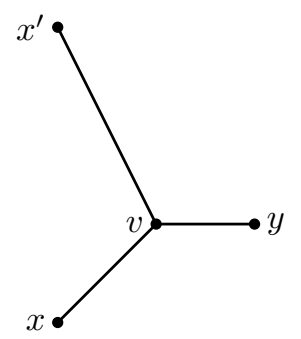

Figure 5. A minimal subtree containing $x, x^{\prime}$ and $y$, with center $v$.

By geodesic stability, Lemma 9, as $v$ lies on the geodesic in $T$ connecting $x$ and $x^{\prime}$, the point $v$ lies within a bounded distance $L_{n}$ of the geodesic in $X$ connecting $x$ and $x^{\prime}$. As $D$ is $Q$-quasiconvex, the geodesic in $X$ from $x$ to $x^{\prime}$ is contained in a $Q$-neighbourhood of $D$, and so

$$
d(D, v) \leqslant Q+L_{n}
$$

As $x$ is a closest point in $D$ to $y, d(x, y)=d(D, y)$, and so the triangle inequality implies

$$
d(x, y) \leqslant d(D, v)+d(v, y)
$$

Using 10 this implies

$$
d(x, y) \leqslant Q+L_{n}+d(v, y) .
$$

As the metric in $X$ is coarsely equivalent to the metric in $T$, using (8) implies

$$
d_{T}(x, y)-n K_{T} \leqslant Q+L_{n}+d_{T}(v, y) .
$$

As $T$ is a tree, $d_{T}(x, y)=d_{T}(x, v)+d_{T}(v, y)$, which gives

$$
d(x, v) \leqslant d_{T}(x, v) \leqslant A,
$$

where $A=Q+L_{n}+n K_{T}$, and so the distance from $x$ to $v$ in $X$, is bounded by a number which only depends on $\delta, Q$ and $n$, the number of points in the approximate tree, as required.

We now prove Proposition 6

Proof. Let $T$ be an approximate tree containing the points $x, x^{\prime}, y$ and $z$, and let $v$ be the center in $T$ for $x, x^{\prime}$ and $y$, and let $w$ be the center in $T$ for $x, y$ and $z$. The set $D$ and the points $x, x^{\prime}$ and $y$ satisfy the conditions of Proposition 10 , and so $d(x, v) \leqslant A_{1}$, where $A_{1}$ depends only on $\delta, Q$ and $n$, and in this case $n$ is equal to 4 .

We now show that condition (2), where $A$ is chosen to be $6 K_{T}+A_{1}$, implies that the centers $v$ and $w$ occur in that order along the geodesic from $x$ to $y$, so the approximate tree $T$ has the configuration illustrated in Figure 6. 


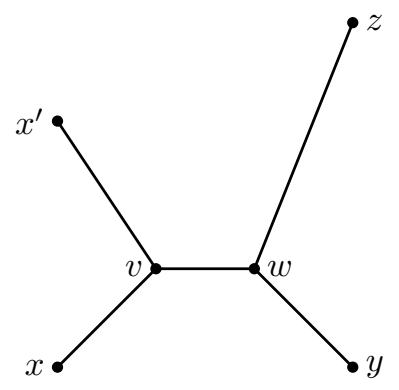

FiguRE 6. An approximate tree containing $x, x^{\prime}, y$ and $z$.

As the metric in $T$ is equal to the metric in $X$ up to bounded additive error, condition (2) is equivalent to

$$
(x \cdot z)_{y}^{T} \leqslant d_{T}(x, y)-A+6 K_{T} .
$$

In $T$, the Gromov product $(x \cdot z)_{y}^{T}$ is equal to $d_{T}(y, w)$, and the center $v$ lies on the geodesic connecting $x$ to $y$, so

$$
d_{T}(w, y) \leqslant d_{T}(x, v)+d_{T}(v, y)-A+6 K_{T} .
$$

As $d(x, v) \leqslant A_{1}$, this implies

$$
d_{T}(w, y) \leqslant d_{T}(v, y)-A+6 K_{T}+A_{1} .
$$

As we have chosen $A=6 K_{T}+A_{1}$, this implies that $d_{T}(w, y) \leqslant d_{T}(v, y)$, and so the centers $v$ and $w$ must lie in that order along the geodesic from $x$ to $y$. The constant $A$ only depends on $\delta, Q$ and $n$, which in this case is 4 , and so $A$ only depends on $\delta$ and $Q$, as required.

In order to show (3), let $x^{\prime}$ be the closest point on $D$ to $z$. Then, given the configuration of the approximate tree shown in Figure 6 .

$$
d_{T}\left(x^{\prime}, z\right) \geqslant d_{T}(v, z) .
$$

As $d_{T}(x, v) \leqslant A_{1}$,

$$
d_{T}\left(x^{\prime}, z\right) \geqslant d_{T}(x, z)-A_{1},
$$

and now using the definition of the Gromov product,

$$
d_{T}\left(x^{\prime}, z\right) \geqslant d_{T}(x, y)+d_{T}(y, z)-2(x \cdot z)_{y}^{T}-A_{1} .
$$

As the metrics in $T$ and $X$ are equal up to additive constants, this implies that

$$
d(D, z) \geqslant d(x, y)+d(y, z)-2(x \cdot z)_{y}-B,
$$

where $B=A_{1}+14 K_{T}$, which only depends on $\delta$ and $Q$, as required.

Finally, the configuration of the approximate tree shown in Figure 6 shows that the two Gromov products $(x \cdot z)_{y}^{T}$ and $\left(x^{\prime} \cdot z\right)_{y}^{T}$ are both equal to $d_{T}(y, w)$, and so 
as the metrics in $T$ and $X$ are equal up to additive error, this shows (4), for some constant $C$, which only depends on $\delta$ and $Q$, as required.

Finally, we prove Proposition 7

Proof. Consider an approximate tree $T$ containing the points $x, x^{\prime}, y, y^{\prime}$ and $z$. Let $v$ be the center in $T$ for $x, x^{\prime}$ and $y$, let $w$ be the center in $T$ for $z, z^{\prime}$ and $y$, and let $u$ be the center for $x, y$ and $z$.

The set $D$ and the points $x, x^{\prime}$ and $y$ satisfy the conditions of Proposition 10 , and so $d(x, v) \leqslant A_{1}$, where $A_{1}$ depends only on $\delta, Q$ and $n$, and in this case $n$ is equal to 5. Similarly, the set $E$ and the points $z, z^{\prime}$ and $y^{\prime}$ also satisfy the conditions of Proposition 10 , and so $d(z, w) \leqslant A_{1}$, where $A_{1}$ depends only on $\delta, Q$ and $n$, and again in this case $n$ is equal to 5 .

We now show that condition (5), where $A$ is chosen to be $15 K_{T} / 2+A_{1}$, implies that the centers $v, u$ and $w$ occur in that order along the geodesic from $x$ to $z$, so the approximate tree $T$ has the configuration illustrated in Figure 7 possibly with some zero length edges.

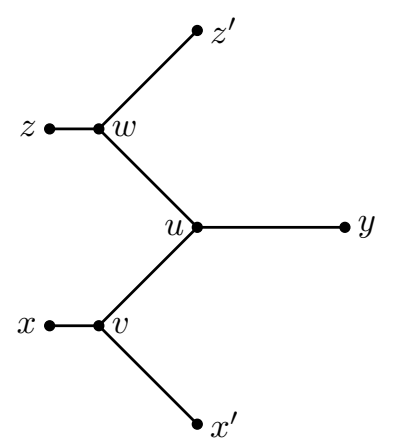

Figure 7. An approximate tree for $x, x^{\prime}, y, z$ and $z^{\prime}$.

As the metric in $T$ is equal to the metric in $X$ up to bounded additive error, condition (5) is equivalent to

$$
(x \cdot z)_{y}^{T} \leqslant \min \left\{d_{T}(x, y), d_{T}(y, z)\right\}-A+15 K_{T} / 2 .
$$

In $T$, the Gromov product $(x \cdot z)_{y}^{T}$ is equal to $d_{T}(y, u)$, and the center $v$ lies on the geodesic in $T$ connecting $x$ to $y$, and the center $w$ lies on the geodesic connecting $z$ to $y$, so

$$
d_{T}(y, u) \leqslant \min \left\{d_{T}(x, v)+d_{T}(v, y), d_{T}(z, w)+d_{T}(w, y)\right\}-A+15 K_{T} / 2 .
$$

As $d(x, v) \leqslant A_{1}$, and $d(z, w) \leqslant A_{1}$, this implies

$$
d_{T}(y, u) \leqslant \min \left\{d_{T}(v, y), d_{T}(w, y)\right\}-A+15 K_{T} / 2+A_{1} .
$$


As we have chosen $A=15 K_{T} / 2+A_{1}$, this implies that $d_{T}(y, u) \leqslant d_{T}(v, y)$, and also $d_{T}(y, u) \leqslant d_{T}(w, y)$, and so the centers $v$ and $w$ must lie further from $y$ than $u$, and the only way in which this can happen is if the center $u$ lies between $v$ and $w$, as illustrated in Figure 7. The constant $A$ only depends on $\delta, Q$ and $n$, which in this case is 5 , and so $A$ only depends on $\delta$ and $Q$, as required.

For estimate (6), the distance $d(D, E)$ between the two quasiconvex sets, suppose that $x^{\prime}$ and $z^{\prime}$ are closest points in $D$ and $E$ respectively, i.e. $d\left(x^{\prime}, z^{\prime}\right)=d(D, E)$. In the approximate tree, $d_{T}\left(x^{\prime}, z^{\prime}\right) \geqslant d_{T}(x, z)$. Using the definition of the Gromov product in the approximate tree gives

$$
d_{T}\left(x^{\prime}, y^{\prime}\right) \geqslant d_{T}(x, y)+d_{T}(y, z)-2(x \cdot z)_{y}^{T}-2 A_{1} .
$$

Now using Lemma 8 to estimate distances in $X$, gives

$$
d(D, E) \geqslant d(x, y)+d(y, z)-2(x \cdot z)_{y}-2 A_{1}-5 K_{T} .
$$

If we choose $B=2 A_{1}+5 K_{T}$, which only depends on $\delta$ and $Q$, then this shows (6).

For the final estimate for the Gromov products, observe that in the approximate tree $T$, the Gromov product $(x \cdot z)_{y}^{T}$ is equal to $\left(x^{\prime} \cdot z^{\prime}\right)_{y}^{T}$, and so again by Lemma 8 .

$$
\left|(x \cdot z)_{y}-\left(x^{\prime} \cdot z^{\prime}\right)_{y}\right| \leqslant 15 K_{T} / 2,
$$

and so we may choose $C=15 K_{T} / 2$, which only depends on $\delta$ and $Q$, and this gives the final estimate (7).

\section{LOCAL ESTIMATES FAR FROM THE DISC SET}

We are interested in estimating distances from points in the curve complex to a particular disc set $\mathcal{D}$. Given a positive number $R$, it will be convenient to consider the following function $\phi_{R}: \mathcal{C}\left(S_{g}\right) \rightarrow \mathbb{N}_{0}$ defined by

$$
\phi_{R}(x)=\lfloor d(\mathcal{D}, x) / R\rfloor,
$$

where for any real number $r$, the function $\lfloor r\rfloor$ is the largest integer less than or equal to $r$. In particular, a random walk on the mapping class group gives rise to a sequence of random variables $X_{n}=\phi_{R}\left(w_{n} x_{0}\right)$, with values in $\mathbb{N}_{0}$.

We now show that if $X_{m}$ is sufficiently large, then the probability that $X_{m+n}$ is less than $X_{m}-r$ decays exponentially in $r$, for all $n$ sufficiently large. As the random walk is a Markov chain on $G$, it suffices to consider the case of a random walk of length $n$ starting at some point $g x_{0}$ in $\mathcal{C}\left(S_{g}\right)$.

Proposition 11. Let $\mu$ be a finitely supported probability distribution on the mapping class group, whose semi-group support $\langle\operatorname{supp}(\mu)\rangle_{+}$is a non-elementary subgroup. For any number $q>0$, there are numbers $R$ and $N$, which depend on $q$ and $\mu$, such that

$$
\mathbb{P}\left(\phi_{R}\left(g w_{n} x_{0}\right) \leqslant t+1-r \mid \phi_{R}\left(g x_{0}\right)=t \geqslant 1\right) \leqslant q^{r}
$$


for all $n \geqslant N$ and $r \geqslant 0$.

Recall that the probability distribution $\mu$ depends on the group $G$, and so any number which depends on $\mu$ implicitly depends on the group $G$, and hence on the coarse geometry constants determined by $G$.

In order to show this, we make use of Proposition 6, which enables us to estimate the distance of $g w_{n} x_{0}$ from a quasiconvex set in terms of the distance travelled by the sample path, and an estimate on the size of a particular Gromov product. We will also use the fact that the distance travelled by the sample path in the curve complex $\mathcal{C}\left(S_{g}\right)$ grows linearly in $n$ with exponential decay.

Theorem 12. Mah12 Let $\mu$ be a probability distribution on the mapping class group with finite support whose semi-group support $\langle\operatorname{supp}(\mu)\rangle_{+}$is a non-elementary subgroup. Then there are numbers $K_{\ell}, L>0$ and $c_{\ell}<1$, which depend on $\mu$, such that

$$
\mathbb{P}\left(d\left(w_{n} x_{0}, x_{0}\right) \leqslant L n\right) \leqslant K_{\ell} c_{\ell}^{n}
$$

for all $n$.

In particular, this result holds when $n=0$, and so the number $K_{\ell}$ must be at least 1. In order to estimate probabilities involving Gromov products, we will also use the following estimate for the probability a sample path lies in a shadow set, as defined in (1), which we shall refer to as exponential decay for shadows.

Lemma 13. Mah12 Let $\mu$ be a probability distribution on the mapping class group with finite support whose semi-group support $\langle\operatorname{supp}(\mu)\rangle_{+}$is a non-elementary subgroup of the mapping class group. Then there are numbers $K_{S}>0$ and $c_{S}<1$, which depend on $\mu$, such that for any point $y$,

$$
\mathbb{P}\left(w_{n} x_{0} \in S_{x_{0}}(y, R)\right) \leqslant K_{S} c_{S}^{d\left(x_{0}, y\right)-R},
$$

for all $n$.

The following estimate for the Gromov product then follows immediately from the definition of shadow sets, (1).

$$
\mathbb{P}\left(\left(y \cdot w_{n} x_{0}\right)_{x_{0}} \geqslant d\left(x_{0}, y\right)-R\right) \leqslant K c^{d\left(x_{0}, y\right)-R} .
$$

In order to simplify notation, we shall unify the exponential decay constants in the two results above, by setting $K=\max \left\{K_{\ell}, K_{S}\right\}$ and $c=\max \left\{c_{\ell}, c_{S}\right\}$.

We now give a brief overview of the proof. Consider a random walk of length $n$ starting at a point $g x_{0}$, reasonably far from the disc set $\mathcal{D}$, and so the endpoint of the random walk is $g w_{n} x_{0}$. By linear progress, it is very likely that the random walk has gone a reasonable distance, and by exponential decay for shadows, it is very unlikely that the Gromov product $\left(x_{0} \cdot g w_{n} x_{0}\right)_{g x_{0}}$ is large, and this Gromov product measures how much the geodesic from $g x_{0}$ to $g w_{n} x_{0}$ fellow-travels, or backtracks, along the path from $g x_{0}$ to $x_{0}$. In this case, we may then apply Proposition 6 to 
estimate the distance from $\mathcal{D}$ to $g w_{n} x_{0}$. This is the main case we consider in the proof below, but we also need to estimate separately the less likely cases in which the random walk does not go very far, or the Gromov product is so large that Proposition 6 does not apply. The final bounds arise from adding the bounds we get in each case.

Proof. (of Proposition 11.) We need to choose appropriate values for $N$ and $R$. In order to make clear that there is no circularity in our choice of constants, we now state how we will choose $N$ and $R$. We shall choose

$$
R \geqslant \max \left\{\log \left(q^{2} / 3 K\right) / \log (c)+A+C, 2 \log \left(q^{2} / 3 K\right) / \log (c), B+2 C\right\},
$$

where $A, B$ and $C$ are the constants from Proposition 6 which only depend on the coarse geometry constants $\delta$ and $Q$, and $K$ and $c$ are the exponential decay constants, which depend on $\mu$. We shall then choose

$$
N \geqslant \max \left\{2 R / L, \log \left(q^{3} / 3 K\right) / \log (c)\right\},
$$

with the same notation for constants as above, and where $L$ is the linear progress constant, which only depends on $\mu$. We emphasize that our choice of $R$ only depends on $q$ and $\mu$, and our choice of $N$ depends on $R, q$ and $\mu$.

We wish to estimate the probability that $\phi_{R}\left(g w_{n} x_{0}\right)$ takes certain values, and so we need to estimate the distance from the disc set $\mathcal{D}$ to $g w_{n} x_{0}$, and in the main case we consider we shall do this by using Proposition 6. We shall apply Proposition 6 with $y=g x_{0}, z=g w_{n} w_{0}$, and $x$ the nearest point in the disc set to $g x_{0}$. Proposition 6 then says that the following condition on the Gromov product

$$
\left(x \cdot g w_{n} x_{0}\right)_{g x_{0}} \leqslant d\left(x, g x_{0}\right)-A,
$$

implies the following bound on the distance from $g w_{n} x_{0}$ to the disc set $\mathcal{D}$,

$$
d\left(\mathcal{D}, g w_{n} x_{0}\right) \geqslant d\left(x, g x_{0}\right)+d\left(g x_{0}, g w_{n} x_{0}\right)-2\left(x \cdot g w_{n} x_{0}\right)_{g x_{0}}-B .
$$

Recall that as $x_{0} \in \mathcal{D}$, the final part of Proposition 6 shows that we may replace $\left(x \cdot g w_{n} x_{0}\right)_{g x_{0}}$ with $\left(x_{0} \cdot g w_{n} x_{0}\right)_{g x_{0}}$ up to bounded error, so 14 is implied by the following condition

$$
\left(x_{0} \cdot g w_{n} x_{0}\right)_{g x_{0}} \leqslant d\left(x, g x_{0}\right)-A-C .
$$

As $d\left(x, g x_{0}\right)=d\left(\mathcal{D}, g x_{0}\right)$, and $d\left(\mathcal{D}, g x_{0}\right) \leqslant R \phi_{R}\left(g x_{0}\right)$, and we have assumed $\phi_{R}\left(g x_{0}\right)=t$, we can rewrite the condition above as

$$
\left(x_{0} \cdot g w_{n} x_{0}\right)_{g x_{0}} \leqslant R t-A-C .
$$

We shall consider various cases, depending on whether some combination of condition (15), and the following condition (16), hold. The second condition is that the sample path has travelled a distance at least $2 R$ from $g x_{0}$ to $g w_{n} x_{0}$, i.e.

$$
d\left(g x_{0}, g w_{n} x_{0}\right) \geqslant 2 R \text {. }
$$


We shall consider the following three cases, defined in terms of the conditions above, which cover all possibilities. The table below summarizes the three cases, the various possibilities for $\phi_{R}\left(g w_{n} x_{0}\right)$ which may occur given the conditions, and the bounds on the probabilities that these values of $\phi_{R}\left(g w_{n} x_{0}\right)$ occur.

$\begin{array}{clll}\text { Case } & \text { Conditions } & \text { Value of } \phi_{R}\left(g w_{n} x_{0}\right) & \text { Probability } \\ 1 & 15) \text { and }(16) \text { hold } & \phi_{R}\left(g w_{n} x_{0}\right) \leqslant t-r & \leqslant q^{r+1} / 3, r \geqslant 1 \\ & & & \leqslant 1, r=0 \\ 2 & 15 \text { fails } & \phi_{R}\left(g w_{n} x_{0}\right) \geqslant 0 & \leqslant q^{t+1} / 3 \\ 3 & 150 \text { fails } & \phi_{R}\left(g w_{n} x_{0}\right) \geqslant t-2 & \leqslant q^{3} / 3\end{array}$

We now consider each case in turn.

Case 1. We first consider the case in which both conditions (15) and (16) hold, and so we may apply Proposition 6. In this case, consequences (3) and (4) imply

$$
d\left(\mathcal{D}, g w_{n} x_{0}\right) \geqslant R t+d\left(g x_{0}, g w_{n} x_{0}\right)-2\left(x_{0} \cdot g w_{n} x_{0}\right)_{g x_{0}}-2 C-B,
$$

where $B$ and $C$ only depend on $\delta$ and $Q$. As condition 16 holds, we are in the case in which $d\left(g x_{0}, g w_{n} x_{0}\right) \geqslant 2 R$, and as we have chosen $R \geqslant B+2 C$ this implies that

$$
d\left(\mathcal{D}, g w_{n} x_{0}\right) \geqslant R t+R-2\left(x_{0} \cdot g w_{n} x_{0}\right)_{g x_{0}},
$$

and so $\phi_{R}\left(g w_{n} x_{0}\right) \leqslant t+1-r$ may only occur if $\left(x_{0} \cdot g w_{n} x_{0}\right)_{g x_{0}} \geqslant R r / 2$. By exponential decay for shadows this happens with probability at most $K c^{R r / 2}$, which is at most $q^{r} / 3$, for $r \geqslant 1$, and at most $K \geqslant 1$ for $r=0$, by 112 , as we have chosen $R \geqslant 2 \log \left(q^{2} / 3 K\right) / \log (c)$. Therefore this case contributes an amount $q^{r} / 3$ to the upper bound for the probability that $\phi_{R}\left(g w_{n} x_{0}\right)=t+1-r$, for all $1 \leqslant r \leqslant t+1$, and an amount 1 to the upper bound for $\phi_{R}\left(g w_{n} x_{0}\right)=t+1$.

Case 1

Case 2. We now consider the case in which condition f15 fails, i.e.

$$
\left(x_{0} \cdot g w_{n} x_{0}\right)_{g x_{0}} \geqslant R t-C-A .
$$

By exponential decay for shadows, Lemma 13 , the probability that condition (17) does not hold is at most $K c^{R t-C-A}$, which is at most $q^{t+1} / 3$, for $t \geqslant 1$, as by (12) we have chosen $R \geqslant \log \left(q^{2} / 3 K\right) / \log (c)+A+C$. In this case there is no restriction on the possible value of $\phi_{R}\left(g w_{n} x_{0}\right)$, and so this case contributes an amount of $q^{t+1} / 3$ to the upper bound for the probability that $\phi_{R}\left(g w_{n} x_{0}\right)=r$ for every possible value of $r \in \mathbb{N}_{0}$.

Case 2

Case 3. We now consider the final case in which the sample path travels distance at most $2 R$, i.e. condition (16) fails. By linear progress with exponential decay, Theorem 12 .

$$
\mathbb{P}\left(d\left(g x_{0}, g w_{n} x_{0}\right) \leqslant L n\right) \leqslant K c^{n} .
$$


By 13. we have chosen $N \geqslant 2 R / L$, and so this implies that

$$
\mathbb{P}\left(d\left(g x_{0}, g w_{n} x_{0}\right) \leqslant 2 R\right) \leqslant K c^{n},
$$

for all $n \geqslant N$. Also by 13 we have chosen $N \geqslant \log \left(q^{3} / 3 K\right) / \log (c)$, and so $K c^{n} \leqslant q^{3} / 3$ for all $n \geqslant N$. As the sample path has not gone very far, then the distance from $\mathcal{D}$ to $g w_{n} x_{0}$ can not have decreased too much. In fact, by the triangle inequality, as

$$
d\left(\mathcal{D}, g w_{n} x_{0}\right) \geqslant d\left(\mathcal{D}, g x_{0}\right)-d\left(g x_{0}, g w_{n} x_{0}\right),
$$

this implies that

$$
d\left(\mathcal{D}, g w_{n} x_{0}\right) \geqslant R t-2 R
$$

i.e. $\phi_{R}\left(g w_{n} x_{0}\right) \geqslant \phi_{R}\left(g x_{0}\right)-2$ in this case, which occurs with probability at most $q^{3} / 3$. This case contributes an amount of $q^{3} / 3$ to the upper bound for those values of $r \in \mathbb{N}_{0}$ satisfying $r \geqslant t-2$.

Case 3

At least one of the three cases above must occur, and so the desired upper bounds arise from summing the probabilities in each case, which we summarize in the table below.

$$
\begin{aligned}
& \text { upper bound } \\
& r \quad \text { Value of } \phi_{R}\left(g w_{n} x_{0}\right)=t+1-r \text { Case } 1 \text { Case } 2 \text { Case } 3 \text { Total } \\
& \begin{array}{ccccc}
4 \leqslant r \leqslant t+1 & 0 \leqslant t+1-r \leqslant t-3 & q^{r} / 3 & +q^{t+1} / 3 & \leqslant q^{r} \\
1 \leqslant r \leqslant 3 & t-2 \leqslant t+1-r \leqslant t & q^{r} / 3 & +q^{t+1} / 3+q^{3} / 3 \leqslant q^{r} \\
r=0 & t+1 \leqslant t+1-r & 1 & +q^{t+1} / 3+q^{3} / 3 \leqslant 1
\end{array}
\end{aligned}
$$

The Total column gives an upper bound on probability that any of the cases occur, which in the final row is the trivial upper bound of 1 .

This completes the proof of Proposition 11.

\section{TRAIN TRACKS AND SHADOWS}

In this section we collect some useful facts about train tracks and shadow sets, as defined in (1). The key observation is that a maximal recurrent train track determines a subset of the curve complex which contains a shadow set.

We briefly review some properties of train tracks, see Penner and Harer PH92 for more details. A train track is a smoothly embedded 1-complex $\tau$ on a surface such that edges (called branches) at each vertex (called a switch) are all tangent, and there is at least one edge in both possible tangent directions at each vertex. Therefore, for each switch, the branches are divided into two non-empty sets of branches with the same signed tangent vector, which are called the incoming and outgoing branches. The complementary regions are surfaces with boundaries and cusps, and none of the complementary regions may be annuli or discs with two or fewer cusps. A train track on a closed surface is maximal if every complementary region is a triangle. 
A train route is a smooth path in $\tau$, and so it crosses a switch by going from an incoming branch to an outgoing branch, or vice versa. A train track is recurrent if every branch is contained in a closed train route. A transverse measure on $\tau$ is a non-negative function on the branches which satisfies the switch condition, i.e. at each switch, the sum of the measures of the incoming branches is equal to the sum of the measures of the outgoing branches. Any closed train route induces a transverse measure on $\tau$ given by the counting measure. We shall write $P(\tau)$ for the collection of transverse measures supported by $\tau$, which is a subset of $\mathcal{M L}$, Thurston's space of measured laminations on the surface, and $P(\tau)$ is a cone on a compact polyhedron in $\mathcal{P} \mathcal{M L}$, the projectivization of $\mathcal{M L}$. If the train track is maximal, then $P(\tau)$ has the same dimension as $\mathcal{M L}$.

A simple closed curve $x$ is carried on $\tau$ if $x$ is homotopic to a train route. A train track $\sigma$ is carried by a train track $\tau$ if every train route on $\sigma$ is homotopic to a train route on $\tau$, and we denote this by $\sigma \prec \tau$.

Given a train track $\tau$, we may produce new train tracks by splitting $\tau$, by the following local modification, illustrated in Figure 8 , in which a subset of the train track corresponding to the top configuration is replaced by one of the three lower configurations.

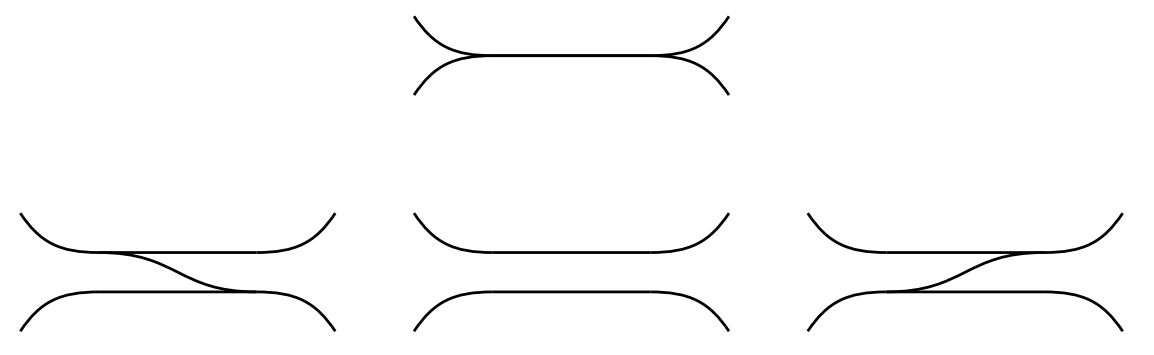

FiguRE 8. Splitting a train track.

The central configuration is called a collision or degenerate split. For our purposes, we will only need to consider non-degenerate splits.

Given a recurrent maximal train track $\tau$, let $C(\tau)$ be the collection of simple closed curves carried by $\tau$.

We start by showing that if two shadow sets intersect, then we may increase the parameter of one of them by a bounded amount such that the new shadow set contains both of the original ones.

Proposition 14. There is a number $R$, which only depends on $\delta$, such that if $S_{x_{0}}\left(x_{1}, R_{1}\right)$ and $S_{x_{0}}\left(x_{2}, R_{2}\right)$ intersect, then $S_{x_{0}}\left(x_{1}, R_{1}\right) \subset S_{x_{0}}\left(x_{2}, \min \left\{d\left(x_{0}, x_{2}\right)-\right.\right.$ $\left.\left.d\left(x_{0}, x_{1}\right)+R_{1}, R_{2}\right\}+R\right)$.

Proof. Let $y$ be a point in the intersection $S_{x_{0}}\left(x_{1}, R_{1}\right) \cap S_{x_{0}}\left(x_{2}, R_{2}\right)$. As $y$ lies in both shadow sets, $\left(x_{1} \cdot y\right)_{x_{0}} \geqslant d\left(x_{1}, y\right)-R_{1}$ and $\left(x_{2} \cdot y\right)_{x_{0}} \geqslant d\left(x_{2}, y\right)-R_{2}$. Recall 
that for any points $x, y$ and $z$, we have

$$
(x \cdot z)_{x_{0}} \geqslant \min \left\{(x \cdot y)_{x_{0}},(y \cdot z)_{x_{0}}\right\}-\delta,
$$

see for example Bridson and Haefliger [BH99, III.H.1.20]. Applying (18) to the three points $x_{1}, x_{2}$ and $y$ implies that $\left(x_{1} \cdot x_{2}\right)_{x_{0}} \geqslant \min \left\{d\left(x_{0}, x_{1}\right)-R_{1}, d\left(x_{0}, x_{2}\right)-R_{2}\right\}-\delta$. Now let $z$ be a point in $S_{x_{0}}\left(x_{1}, R_{1}\right)$, so $\left(x_{1} \cdot z\right)_{x_{0}} \geqslant d\left(x_{0}, z\right)-R_{1}$. Similarly, applying (18) to the three points $x_{1}, x_{2}$ and $z$ implies that $\left(x_{2} \cdot z\right)_{x_{0}} \geqslant \min \left\{d\left(x_{0}, x_{1}\right)-\right.$ $\left.R_{1}, d\left(x_{0}, x_{2}\right)-R_{2}\right\}-2 \delta$, so we may choose $R=2 \delta$.

A shadow set is always non-empty as long as $R \geqslant 0$, and furthermore, if the parameter $R$ is sufficiently large, the shadow sets have non-empty limit sets in $\partial \mathcal{C}\left(S_{g}\right)$. We say a group $G$ acts coarsely transitively on $X$ if there is a number $K$ such that for any $x$ and $y$ in $X$, there is a group element $g$ such that $d(g x, y) \leqslant K$. The action of $\operatorname{MCG}\left(S_{g}\right)$ on $\mathcal{C}\left(S_{g}\right)$ is coarsely transitive.

Proposition 15. BHM11 Let $X$ be a Gromov hyperbolic space, which need not be locally compact, but whose isometry group acts coarsely transitively on $X$. There is a number $R_{0} \geqslant 0$, which only depends on $\delta$, such that for all $R \geqslant R_{0}$ the shadow set $S_{x_{0}}(x, R)$ has a limit set in $\partial X$ which contains a non-empty open set, for all $x$ and $x_{0}$.

For any number $A>0$, the shadow set $S_{x_{0}}(x, R)$ is contained in $S_{x_{0}}(x, R+A)$, and furthermore, if $A$ is sufficiently large, then the limit sets of the shadow sets are strictly nested, and the distance between them in $\mathcal{C}\left(S_{g}\right)$ is bounded below in terms of $A$.

Lemma 16. Mah12 Let $X$ be a $\delta$-hyperbolic space, which need not be locally compact. There is a number $K$, which depends only on $\delta$, such that for all positive numbers $A$ and $R$, and any $x, y \in X$ with $d(x, y) \geqslant A+R+2 K$, the closure of the shadow $S_{x}(y, R)$ is disjoint from the closure of the complement of the shadow $S_{x}(y, R+A+K)$, i.e.

$$
\overline{S_{x}(y, R)} \cap \overline{X \backslash S_{x}(y, R+A+K)}=\varnothing .
$$

Furthermore for any pair of points $a, b \in X$ such that $a \in S_{x}(y, R)$ and $b \in X \backslash$ $S_{x}(y, R+A+K)$, the distance between $a$ and $b$ is at least $A$.

We will use these two properties in the form of the following elementary corollary, which says that every shadow set contains shadow sets nested inside it by an arbitrarily large distance.

Corollary 17. There is a number $R_{0} \geqslant 0$ such that for any number $A \geqslant 0$, and for any shadow set $S_{x}\left(y, R_{0}\right)$, there is a shadow set $S_{x}\left(z, R_{0}\right) \subset S_{x}\left(y, R_{0}\right)$ with $\overline{\mathcal{C}\left(S_{g}\right) \backslash S_{x}\left(y, R_{0}\right)} \cap \overline{S_{x}\left(z, R_{0}\right)}=\varnothing$ and $d\left(\mathcal{C}\left(S_{g}\right) \backslash S_{x}\left(y, R_{0}\right), S_{x}\left(z, R_{0}\right)\right) \geqslant A$.

We also observe that the complement of a shadow set is roughly a shadow set. 
Lemma 18. Mah12 There is a number $K$, which only depends on $\delta$, such that for all numbers $R \geqslant 2 K$, and all $x, z \in \mathcal{C}\left(S_{g}\right)$ with $d(x, z) \geqslant R+K$,

$$
S_{x}(z, d(x, z)-R-K) \subset \mathcal{C}\left(S_{g}\right) \backslash S_{z}(x, R) \subset S_{x}(z, d(x, z)-R+K) .
$$

Finally, we recall the following "change of basepoint" result for shadow sets.

Lemma 19. Mah12 There are numbers $A$ and $B$, which only depend on $\delta$, such that for any $r$, and any three points $x, y$ and $z$ with $(x \cdot y)_{z} \leqslant r-A$, there is an inclusion of shadows, $S_{z}(x, r) \subset S_{y}(x, s)$, where $s=d(x, y)-d(x, z)+r-B$.

We now provide a link between subsets of the curve complex determined by train tracks and shadow sets, by showing that every maximal train track $\tau$ determines a subset $C(\tau)$ of the curve complex which contains a shadow set.

We shall write $\mathcal{L}_{\text {min }}\left(S_{g}\right)$ for the set of all laminations corresponding to minimal foliations, i.e. those laminations which neither contain simple closed curves, and are not disjoint from any simple closed curves. This is a subset of $\mathcal{P} \mathcal{M L}\left(S_{g}\right)$ with the relative topology. The set of ending laminations $\mathcal{E} \mathcal{L}\left(S_{g}\right)$ is a quotient of $\mathcal{L}_{\text {min }}\left(S_{g}\right)$ by the equivalence relation of topological equivalence, i.e. two measured laminations are identified if they correspond to different measures on the same topological lamination. We shall write $\mathcal{T}\left(S_{g}\right)$ for the Teichmüller space of the surface $S_{g}$, which is the space of hyperbolic metrics on the surface. We shall write $\iota$ for the coarsely well-defined map $\iota: \mathcal{T}\left(S_{g}\right) \rightarrow \mathcal{C}\left(S_{g}\right)$, which sends a point in Teichmüller space to a simple closed curve on the surface of shortest length with respect to the corresponding metric. Klarreich Kla, see also Hamenstädt Ham06, showed the Gromov boundary of the complex of curves is homeomorphic to the space of ending laminations.

Theorem 20. Kla The inclusion map $\iota: \mathcal{T}\left(S_{g}\right) \rightarrow \mathcal{C}\left(S_{g}\right)$ extends continuously to the portion $\mathcal{L}_{\text {min }}\left(S_{g}\right)$ of minimal laminations of $\mathcal{P} \mathcal{M L}\left(S_{g}\right)$ to give a map $\pi: \mathcal{L}_{\text {min }}\left(S_{g}\right) \rightarrow$ $\partial \mathcal{C}\left(S_{g}\right)$. The map $\pi$ is surjective and $\pi(\mathcal{L})=\pi\left(\mathcal{L}^{\prime}\right)$ if and only if $\mathcal{L}$ are topologically equivalent, and in fact $\pi$ induces a homeomorphism between $\mathcal{E} \mathcal{L}\left(S_{g}\right)$ and $\partial \mathcal{C}\left(S_{g}\right)$.

We now use this to show that every maximal recurrent train track $\tau$ determines a subset $C(\tau)$ of the complex of curves which contains a shadow set.

Proposition 21. For any simple closed curve $x$ and any maximal recurrent train track $\tau$ there is a simple closed curve $y$ and a number $R \geqslant R_{0}$ such that the shadow set $S_{x}(y, R)$ is contained in $C(\tau)$.

Proof. Let $S_{x}(y, R)$ be any shadow set with $R \geqslant R_{0}$, with $d(x, y)$ sufficiently large such that the closure of $S_{x}(y, R)$ is not equal to all of $\mathcal{C}\left(S_{g}\right)$.

Given a pseudo-Anosov element $\phi$ in $\operatorname{MCG}\left(S_{g}\right)$, we shall write $\left(\mathcal{L}^{s}(\phi), \mathcal{L}^{u}(\phi)\right)$ for the pair in $\mathcal{P} \mathcal{M L} \times \mathcal{P} \mathcal{M L}$ consisting of its stable and unstable laminations. 
Such pairs $\left(\mathcal{L}^{s}(\phi), \mathcal{L}^{u}(\phi)\right)$ are dense in $\mathcal{P} \mathcal{M L} \times \mathcal{P} \mathcal{M L}$, as $\phi$ runs over all pseudoAnosov elements in $\operatorname{MCG}\left(S_{g}\right)$, see for example Mah10, Lemma 3.4]. Furthermore, a pseudo-Anosov element $\phi$ acts with north-south dynamics on the Thurston compactification $\mathcal{T}\left(S_{g}\right) \cup \mathcal{P} \mathcal{M} \mathcal{L}\left(S_{g}\right)$, with fixed points the stable and unstable laminations $\mathcal{L}^{s}(\phi)$ and $\left.\mathcal{L}^{u}(\phi)\right)$ of $\phi$. This means that for any open set $U$ containing $\mathcal{L}^{s}(\phi)$ and disjoint from $\mathcal{L}^{u}(\phi)$, and for any closed set $V$ disjoint from the fixed points, there is a number $N$, depending on $U, V$ and $g$ such that $g^{n}(V) \subset U$ for all $n \geqslant N$.

Therefore, it suffices to show that there is a shadow set $S_{x}(y, R)$, such that the closure of $\iota^{-1}\left(S_{x}(y, R)\right)$ in the Thurston compactification $\mathcal{T}\left(S_{g}\right) \cup \mathcal{P} \mathcal{M L}\left(S_{g}\right)$ is disjoint from an open set in $\mathcal{P} \mathcal{M L}$. Suppose not, then the closure of $\iota^{-1}\left(S_{x}(y, R)\right)$ is dense in $\mathcal{P} \mathcal{M L}$. As the ending laminations $\mathcal{E} \mathcal{L}$ are dense in $\mathcal{P} \mathcal{M L}$, and their image under $\iota$ is equal to $\partial \mathcal{C}\left(S_{g}\right)$, this implies that the closure of the shadow $S_{x}(y, R)$ is equal to all of $\partial \mathcal{C}\left(S_{g}\right)$, a contradiction.

We now show that if the closure of a shadow set $S_{x}(y, R)$ contains a limit point of the subgroup supporting the random walks, then there is a slightly larger shadow set $S_{x}\left(y, R+R_{1}\right)$ whose closure has positive measure with respect to the hitting measure $\nu$.

Proposition 22. There is a number $R_{1}>0$ such that if $S_{x_{0}}(y, R)$ contains a limit point of $H$, then $\nu\left(\overline{S_{x_{0}}\left(y, R+R_{1}\right)}\right)>0$. Furthermore, for any number $D$, there is a shadow set $S_{x_{0}}\left(z, R_{0}\right) \subset S_{x_{0}}\left(y, R+R_{1}\right)$, distance at least $D$ from $x_{0}$, with $\nu\left(\overline{S_{x_{0}}\left(z, R_{0}\right)}\right)>0$.

Proof. There is a sequence of group elements $\left(h_{i}\right)_{i \in \mathbb{N}}$ such that $\left(h_{i} x_{0}\right)_{i \in \mathbb{N}}$ converges to $\mathcal{L}$ in $S_{x_{0}}(y, R)$, so infinitely many of the $\left(h_{i} x_{0}\right)_{i \in \mathbb{N}}$ lie in $S_{x_{0}}\left(y, R+R_{1}\right)$. Choose one with $\mu_{n}\left(h_{i}\right)>0$ for some $n$. Then by exponential decay for shadows, a definite proportion of sample paths starting from $h_{i} x_{0}$ at time $n$ converge into $S_{x_{0}}(y, R+$ $\left.R_{2}\right)$, so $\nu\left(\overline{S_{x_{0}}\left(y, R+R_{2}\right)}\right)>0$, as required.

For any $D$, the countable collection of sets $S_{x_{0}}\left(z, R_{0}\right)$, as $z$ runs over all vertices of the curve complex $\mathcal{C}\left(S_{g}\right)$ with $d\left(x_{0}, z\right) \geqslant D$, cover $S_{x_{0}}\left(y, R+R_{2}\right)$, so at least one of these has positive measure, and is contained in $S_{x_{0}}\left(y, R+R_{3}\right)$, for some $R_{3}$.

\section{Local eStimates Close to THE DisC SET}

The main purpose of this section is to show

Proposition 23. For any complete subgroup $G<M C G\left(S_{g}\right)$ there is a finitely generated non-elementary subgroup $H<G$, such that any finitely supported probability distribution $\mu$, whose semi-group support $\langle\operatorname{supp}(\mu)\rangle_{+}$is a subgroup containing $H$, has the following property.

For any number $R$ there are numbers $N$ and $\varepsilon>0$, depending only on $R$ and $\mu$, such that for any mapping class group element $g$, there is a definite probability $\varepsilon$ 
that the random walk of length $n$ generated by $\mu$ starting at $g$ is distance at least $R$ from the disc set $\mathcal{D}$, so in particular

$$
\mathbb{P}\left(\phi_{R}\left(g w_{n} x_{0}\right)=1 \mid \phi_{R}\left(g x_{0}\right)=0\right) \geqslant \varepsilon,
$$

for all $n \geqslant N$

We will construct such a subgroup $H$ using the following result of Kerckhoff Ker90.

Theorem 24. Ker90 Proposition on p36] There is a recurrent maximal train track $\tau$ on a closed orientable surface $S_{g}$, such that for any identification of $S_{g}$ with the boundary of a handlebody, $\tau$ can be split at most $-9 \chi\left(S_{g}\right)$ times to a recurrent maximal train track $\tau^{\prime}$ such that $N\left(\tau^{\prime}\right)$ is disjoint from the disk set $\mathcal{D}$ of the handlebody. Here $\chi\left(S_{g}\right)$ is the Euler characteristic of the surface.

We now prove Proposition 23 .

Proof. (of Proposition 23, ) Let $\tau$ be a recurrent maximal train track, satisfying Theorem 24, and let $T$ be the finite collection of maximal train tracks obtained by splitting $\tau$ at most $-9 \chi\left(S_{g}\right)$ times.

By Proposition 21, the subset of the curve complex $C\left(\tau_{i}\right)$ corresponding to each maximal train track $\tau_{i} \in T$ contains a shadow set which we shall denote $S_{i}=$ $S_{x_{0}}\left(y_{i}, R_{0}\right)$. Furthermore, for any number $R \geqslant 0$, for each $S_{i}$ we may choose a nested shadow set $S_{i}^{\prime}=S_{x_{0}}\left(y_{i}^{\prime}, R_{0}\right)$, with $\overline{S_{i}^{\prime}} \cap \overline{S_{i} \backslash \mathcal{C}\left(S_{g}\right)}=\varnothing$ and $d\left(\mathcal{C}\left(S_{g}\right) \backslash S_{i}, S_{i}^{\prime}\right) \geqslant R$, for each $i$.

The subgroup $G$ is complete in $\operatorname{MCG}\left(S_{g}\right)$, and so endpoints of pseudo-Anosov elements are dense in $\partial \mathcal{C}\left(S_{g}\right)$. In fact, the pairs $\left(F_{+}, F_{-}\right)$of stable and unstable laminations are dense in $\partial \mathcal{C}\left(S_{g}\right) \times \partial \mathcal{C}\left(S_{g}\right)$. Each shadow set $S_{i}^{\prime}$ contains a nonempty open set in $\partial \mathcal{C}\left(S_{g}\right)$, so for each $S_{i}^{\prime}$, choose a pseudo-Anosov element $g_{i}$, at least one of whose limit points lies in $S_{i}^{\prime}$. Let $H$ be the finitely generated subgroup generated by the finite list of elements $g_{i}$.

By Proposition 22, for any finitely supported probability distribution $\mu$ whose semi-group support $\langle\operatorname{supp}(\mu)\rangle_{+}$is a group containing $H$, the hitting measure $\nu\left(\overline{S_{i}}\right)>$ 0 . Set $\varepsilon=\min \nu\left(\overline{S_{i}}\right) / 2$. As the convolution measures $\mu_{n}$ weakly converge to $\nu$, there is an $N$ such that $\mu_{n}\left(\overline{S_{i}}\right) \geqslant \varepsilon$ for all $n \geqslant N$ and for all $i$.

Now consider a random walk of length $n$ starting from $g x_{0}$. We wish to estimate the distance $d\left(\mathcal{D}, g w_{n} x_{0}\right)$, and by applying the isometry $g^{-1}$, this is equivalent to considering the distance $d\left(g^{-1} \mathcal{D}, w_{n} x_{0}\right)$. By Theorem 24, for any disc set, in particular the disc set $g^{-1} \mathcal{D}$, there is some maximal train track $\tau_{i}$ in $T$ disjoint from $g^{-1} \mathcal{D}$, and so if $w_{n} x_{0}$ lies in the nested shadow set $S_{i}^{\prime}$ contained in $C\left(\tau_{i}\right)$, then $d\left(g^{-1} \mathcal{D}, w_{n} x_{0}\right) \geqslant R$, and so $\phi_{R}\left(g w_{n} x_{0}\right) \geqslant 1$. As $\mu_{n}\left(S_{i}^{\prime}\right) \geqslant \varepsilon$ for all $n \geqslant N$, this implies that $w_{n} x_{0}$ lies in $S_{i}^{\prime}$ with probability at least $\varepsilon$, and so this completes the proof of Proposition 23 . 


\section{EXPONENTIAL DECAY FOR DISTANCE FROM THE DISC SET}

We now use the local estimates for distance from the disc set obtained in the previous sections to show that the distance from $w_{n} x_{0}$ to the disc set grows linearly with exponential decay.

Proposition 25. For any complete subgroup $G$ of $M C G\left(S_{g}\right)$, there is a finitely generated subgroup $H<G$, such that for any finitely supported probability distribution $\mu$ whose semi-group support $\langle\operatorname{supp}(\mu)\rangle_{+}$is a subgroup containing $H$, there are numbers $K, L>0$ and $c<1$, which depend on $\mu$, such that for any disc set $\mathcal{D}$ and any basepoint $x_{0}$, the distance of $w_{n} x_{0}$ from the disc set $\mathcal{D}$ grows linearly with exponential decay, i.e.

$$
\mathbb{P}\left(d\left(\mathcal{D}, w_{n} x_{0}\right) \leqslant L n\right) \leqslant K c^{n} .
$$

We show this by comparing the distribution of the random variables $d\left(\mathcal{D}, w_{n} x_{0}\right)$ with a Markov chain on $\mathbb{N}_{0}$ which gives an upper bound on the probability that the random variable takes small values. Ultimately, we show the Markov chain $\left(\mathbb{N}, P_{0}\right)$ has spectral radius $\rho(P)$ strictly less than 1 , and this gives the exponential decay result we require.

One minor technical point is that the local estimates hold for all $n \geqslant N$. We now observe that if an exponential decay estimate holds for the iterated random walk $w_{n N}$ generated by the $N$-fold convolution measure $\mu_{N}$, then a similar exponential decay estimate holds for $w_{n}$, though for different constants.

Proposition 26. Let $\mu$ be a finitely supported probability distribution such that for some $N>0$, there are numbers $K, L>0$ and $c<1$ such that

$$
\mathbb{P}\left(d\left(\mathcal{D}, w_{n N} x_{0}\right) \leqslant L n\right) \leqslant K c^{n}
$$

Then there are numbers $L^{\prime}>0$ and $c^{\prime}<1$ such that

$$
\mathbb{P}\left(d\left(\mathcal{D}, w_{n} x_{0}\right) \leqslant L^{\prime} n\right) \leqslant K c^{\prime n}
$$

for all $n$.

Proof. As $\mu$ has finite support, with diameter $R$ say, $d\left(\mathcal{D}, w_{n N+k} x_{0}\right) \leqslant d\left(\mathcal{D}, w_{n N} x_{0}\right)+$ $R N$, for any $0 \leqslant k \leqslant N$, so we may choose $L^{\prime}=L+R N$ and $c^{\prime}=c^{1 / N}$.

This shows that by replacing $\mu$ with $\mu_{N}$, we may assume that the local estimates hold for $N=1$, and we shall do this for the remainder of this section to simplify notation. We now consider the sequence of random variables $X_{n}: \Omega \rightarrow \mathbb{N}_{0}$ defined by $X_{n}(\omega)=\phi_{R}\left(w_{n} x_{0}\right)$.

We wish to compare the distributions of the $X_{n}$ with the distributions $X_{n}^{\prime}$ arising from the following Markov chain $\left(\mathbb{N}_{0}, P\right)$, starting with total mass 1 at $0 \in \mathbb{N}_{0}$ at 
time $n=0$. We shall write $p(i, j)$ for the probability you go from $i$ to $j$.

$$
p(0, j)=\left\{\begin{array}{cc}
1-\varepsilon & \text { if } j=0 \\
\varepsilon & \text { if } j=1 \\
0 & \text { if } j \geqslant 2
\end{array}\right.
$$

and for $i>0$,

$$
p(i, j)=\left\{\begin{array}{cl}
q^{i-j+1} & \text { if } j \leqslant i \\
1-q-q^{2}-\cdots q^{i+1} & \text { if } j=i+1 \\
0 & \text { if } j \geqslant i+2
\end{array}\right.
$$

Figure 2 illustrates the first few vertices of this Markov chain.

Given a probability measure $P$ on $\mathbb{N}_{0}$, we shall write $F_{P}$ for the cumulative distribution function of $X$, i.e.

$$
F_{P}(n)=\sum_{i=0}^{n} P(i)
$$

Given two probability measures $P$ and $P^{\prime}$ on $\mathbb{N}_{0}$, we say that $P^{\prime}$ stochastically dominates $P$ if $F_{P}(n) \geqslant F_{P^{\prime}}(n)$ for all $n$, and we shall denote this by $P \preccurlyeq P^{\prime}$. Similarly, given a random variable $X$ which takes values in $\mathbb{N}_{0}$, i.e. $X:(I, \mathbb{P}) \rightarrow \mathbb{N}_{0}$, we shall write $F_{X}$ for the cumulative distribution function of $X$. We say a random variable $X$ stochastically dominates a random variable $X^{\prime}$ if $F_{X}(n) \leqslant F_{X^{\prime}}(n)$ for all $n$, and we shall denote this by $X^{\prime} \preccurlyeq X$.

Let $\left\{X_{n}\right\}$ be a sequence of random variables with values in $\mathbb{N}_{0}$. The transition kernels for the sequence are the measures on $\mathbb{N}_{0}$ given by

$$
K_{n}\left(i_{1}, \ldots, i_{n-1}\right)(A)=\mathbb{P}\left(X_{n} \in A \mid\left(X_{1}, \ldots, X_{n-1}\right)=\left(i_{1}, \ldots i_{n-1}\right)\right),
$$

where $A \subset \mathbb{N}_{0}$. If the transition kernels for two sequences of random variables $\left\{X_{n}\right\}$ and $\left\{X_{n}^{\prime}\right\}$ satisfy $K_{n}^{\prime}\left(i_{1}, \ldots i_{n-1}\right) \preccurlyeq K_{n}\left(i_{1}, \ldots i_{n-1}\right)$ for all $n$ and $i_{1}, \ldots i_{n-1}$, then in fact $X_{n}^{\prime} \preccurlyeq X_{n}$ for all $n$. We now state a precise form of this result, which is often referred to as Strassen's Theorem, or the Stochastic Domination Theorem, see Lindvall [Lin92, Chapter IV]. This result holds in much greater generality, and the version we state here is a simplified one sufficient for our purposes.

Theorem 27. Lin92, Theorem 5.8] Let $\left\{X_{n}\right\}$ and $\left\{X_{n}^{\prime}\right\}$ be sequences of random variables with values in $\mathbb{N}_{0}$ such that $X_{0}^{\prime} \preccurlyeq X_{0}$, and $K_{n}^{\prime}\left(i_{1}, \ldots i_{n-1}\right) \preccurlyeq K_{n}\left(i_{1}, \ldots i_{n-1}\right)$, for all $n$ and $i_{1}, \ldots i_{n-1}$. Then

$$
X_{n}^{\prime} \preccurlyeq X_{n},
$$

for all $n$.

As in our case $X_{0}^{\prime}=X_{0}$, in order to show that $X_{n}^{\prime} \preccurlyeq X_{n}$, it suffices to show that the transition kernels satisfy $K_{n}^{\prime} \preccurlyeq K_{n}$, and we show this using the local estimates, Propositions 11 and 23 . 
Proposition 28. For all $n$, and all $i_{1}, \ldots, i_{n-1}$, the transition kernels for $\left\{X_{n}\right\}$ and $\left\{X_{n}^{\prime}\right\}$ satisfy

$$
K_{n}^{\prime}\left(i_{1}, \ldots, i_{n-1}\right) \preccurlyeq K_{n}\left(i_{1}, \ldots, i_{n-1}\right) .
$$

Proof. As the random variables $\left\{X_{n}^{\prime}\right\}$ arise from a Markov chain, $K_{n}^{\prime}\left(i_{1}, \ldots, i_{n-1}\right)$ only depends on the value of $i_{n-1}$, and may be computed from the defining transition probabilities $p(i, j)$ of the Markov chain.

We first consider the case in which $i_{n-1}=0$. By Proposition 23.

$$
\mathbb{P}\left(X_{n}(\omega)=0 \mid X_{n-1}(\omega)=0\right) \leqslant 1-\varepsilon,
$$

for all $n$, and writing this out in terms of the cumulative probability distributions gives

$$
F_{K_{n}\left(i_{1}, \ldots, i_{n-2}, 0\right)}(0) \leqslant F_{K_{n}^{\prime}\left(i_{1}, \ldots i_{n-2}, 0\right)}(0) .
$$

By definition of the Markov chain, the value of $X_{n}^{\prime}$ may not increase by more than one from the value of $X_{n-1}^{\prime}$, and so $F_{K_{n}^{\prime}\left(i_{1}, \ldots i_{n-2}, 0\right)}(1)=1$, and so this shows that

$$
K_{n}^{\prime}\left(i_{1}, \ldots i_{n-2}, 0\right) \preccurlyeq K_{n}\left(i_{1}, \ldots i_{n-2}, 0\right)
$$

for all $n$ and all $i_{1}, \ldots, i_{n-2}$.

We now consider the case in which $i_{n-1}>0$. By Proposition 11

$$
\mathbb{P}\left(X_{n}(\omega)=k-i \mid X_{n-1}(\omega)=k\right) \leqslant q^{i+1},
$$

for all $0 \leqslant i \leqslant k$, and for all $n$, and so by summing over values of $i$ with $l \leqslant i \leqslant k$, this implies that

$$
F_{K_{n}\left(i_{1}, \ldots i_{n-2}, k\right)}(l) \leqslant F_{K_{n}^{\prime}\left(i_{1}, \ldots i_{n-2}, k\right)}(l),
$$

for all $l \leqslant k$. Again, by definition of the Markov chain, the value of $X_{n}^{\prime}$ may not increase by more than 1 at any step, and so $F_{K_{n}^{\prime}\left(i_{1}, \ldots i_{n-2}, k\right)}(k+1)=1$, and so this shows that

$$
K_{n}^{\prime}\left(i_{1}, \ldots i_{n-2}, k\right) \preccurlyeq K_{n}\left(i_{1}, \ldots i_{n-2}, k\right),
$$

for all $n$ and all $i_{1}, \ldots i_{n-2}$, and with $k>0$.

We now show that the Markov chain $\left(\mathbb{N}_{0}, P\right)$ has spectral radius $\rho(P)<1$. If $f$ is a function on $\mathbb{N}_{0}$ we shall write $P f$ to denote the function $\operatorname{Pf}(k)=\sum p(k, j) f(j)$.

Proposition 29. If $q<1 / 4$ then the Markov chain $\left(\mathbb{N}_{0}, P\right)$ has spectral radius $\rho<1$.

Proof. Recall from Woess Woe00, Section 7] that $\rho \leqslant t$ if there is a strictly positive $t$-superharmonic function $f$ on $\mathbb{N}_{0}$, i.e the function $f$ satisfies $f(k)>0$ for each $k$, and $P f \leqslant t f$. We will show that if we choose $t=\max \{1-\varepsilon(1-2 q), 4 q\}$, then the function $f(k)=(2 q)^{k}$ is $t$-superharmonic for $t<1$ as long as $q<1 / 4$. This may be verified by an elementary calculation, and we provide the details below for the convenience of the reader. 
The first inequality $\operatorname{Pf}(k) \leqslant t f(k)$, for $k=0$, is

$$
(1-\varepsilon) f(0)+\varepsilon f(1) \leqslant t f(0),
$$

and then the remaining inequalities are of the form

$$
q^{k+1} f(0)+q^{k} f(1)+\cdots+q f(k)+p_{k} f(k+1) \leqslant t f(k),
$$

for $k \geqslant 1$, where $p_{k}=1-q-q^{2}-\cdots q^{k+1}$.

The first inequality (21), gives

$$
1-\varepsilon(1-2 q) \leqslant t,
$$

which is satisfied for some $t<1$ if $q<1 / 2$.

For the general case 22 , we obtain

$$
q^{k+1}+q^{k}(2 q)+\cdots+q(2 q)^{k}+p_{k}(2 q)^{k+1} \leqslant t(2 q)^{k} .
$$

As $p_{k} \leqslant 1$, this inequality is satisfied if

$$
q^{k+1}\left(1+2+\cdots 2^{k}\right)+(2 q)^{k+1} \leqslant t(2 q)^{k} .
$$

As the sum of the geometric series is less than $2^{k+1}$, it suffices to choose $q$ such that

$$
4 q \leqslant t
$$

and this holds for some $t<1$ if $q<1 / 4$, as required.

We shall write $p^{(n)}(i, j)$ for the probability that the Markov chain starting at $i$ at time 0 is at location $j$ on the $n$th step. A Markov chain on a graph is uniformly irreducible if there are numbers $N$ and $\varepsilon_{0}$ such that for any pair of neighbouring vertices $i$ and $j, p^{(n)}(x, y) \geqslant \varepsilon_{0}$ for some $n \leqslant N$. If we consider $\mathbb{N}_{0}$ to have the standard graph structure in which $i$ and $j$ are connected by an edge if and only if $|i-j| \leqslant 1$, then the Markov chain $\left(\mathbb{N}_{0}, P\right)$ above is uniformly irreducible, with $N=1$ and $\varepsilon_{0}=\min \left\{\varepsilon, q^{2}, 1-q /(1-q)\right\}$.

Lemma 30. Woe00, Lemma 8.1] If the Markov chain $(X, P)$ is uniformly irreducible, then there is a number $A>0$ such that $p^{(n)}(x, y) \leqslant A^{d(x, y)} \rho(P)^{n}$.

Therefore, by Lemma 30 , there is a number $A>0$ such that $F_{X_{n}}(L n) \leqslant$ $L n A^{L n} \rho^{n}$, and this decays exponentially for some $L>0$, chosen sufficiently close to zero.

\section{Exponential Decay for HeegaArd Splitting Distance}

In this section we prove linear progress with exponential decay for Heegaard splitting distance, Theorem 5 , using linear progress with exponential decay for distance from the disc set, Proposition 25, and Proposition 75 to estimate the distance between two quasiconvex sets. 
Proof. (of Theorem 5) It will be convenient to think of the sample path $w_{n}$ as the concatenation of two sample paths of lengths roughly $n / 2$. To be precise, choose $m=\lfloor n / 2\rfloor$. We may then consider the sample path $w_{n}$ to consist of two segments, an initial segment $w_{m}$ of length $m$, and a final segment $w_{m}^{-1} w_{n}$, of length $n-m$. These two sample paths $w_{m}$ and $w_{m}^{-1} w_{n}$ are independently distributed.

The distance from $\mathcal{D}$ to $w_{m} x_{0}$ decays exponentially in $n$, by Proposition 25, i.e.

$$
\mathbb{P}\left(d\left(\mathcal{D}, w_{m} x_{0}\right) \leqslant L n / 2\right) \leqslant K c^{n / 2} .
$$

Proposition 25 also applies to the reflected random walk generated by the probability distribution $\check{\mu}(g)=\mu\left(g^{-1}\right)$, with the same basepoint $x_{0}$, but with the disc set $h_{S^{3}} \mathcal{D}$, though possibly with different numbers $\check{K}, \check{L}>0$ and $\check{c}<1$. To simplify notation, we shall write $\mathcal{D}^{\prime}$ for $h_{S^{3}} \mathcal{D}$. Therefore applying Proposition 25 to the reflected random walk of length $n-m$ gives

$$
\mathbb{P}\left(d\left(\mathcal{D}^{\prime},\left(w_{m}^{-1} w_{n}\right)^{-1} x_{0}\right) \leqslant \check{L}(n / 2+1)\right) \leqslant \check{K} \check{c} \check{c}^{n / 2+1} .
$$

Now applying the isometry $w_{n}$ we obtain

$$
\mathbb{P}\left(d\left(w_{m} x_{0}, w_{n} \mathcal{D}^{\prime}\right) \leqslant \check{L}(n / 2+1)\right) \leqslant \check{K} \check{c}^{n / 2+1} .
$$

Choose $L_{1}=\min \{L, \check{L}\}$, and let $y$ be a closest point in $\mathcal{D}$ to $w_{m} x_{0}$, and similarly, let $y^{\prime}$ be a closest point in $\mathcal{D}^{\prime}$ to $w_{m} x_{0}$. We may apply Proposition 7 , with the quasiconvex sets chosen to be the two discs sets, and $y$ chosen to be $w_{m} x_{0}$, unless condition (5) fails, which in this context is

$$
\left(y \cdot y^{\prime}\right)_{w_{m} x_{0}} \leqslant L_{1}(n / 2+1)-A,
$$

where $A$ is the constant from Proposition 7 However, we now show that the probability that this condition fails decays exponentially in $n$. If we choose $N=$ $4 A / L_{1}$, then $L_{1}(n / 2+1)-A \geqslant L_{1} n / 4-C-B / 2$, for all $n \geqslant N$, where $A, B$ and $C$ are the constants from Proposition 7 . Using the Gromov product estimate (7) from Proposition 7, and exponential decay for shadows, there are numbers $K_{1}$ and $c_{1}$ such that

$$
\mathbb{P}\left(\left(x_{0} \cdot w_{n} x_{0}\right)_{w_{m} x_{0}} \geqslant L_{1} n / 4-C-B / 2\right) \leqslant K_{1} c_{1}^{L_{1} n / 4-C-B / 2} .
$$

Assuming (25) fails, Proposition 7 line $(6)$ implies that the distance from $\mathcal{D}$ to $w_{n} \mathcal{D}^{\prime}$ is at least

$$
d\left(\mathcal{D}, w_{n} \mathcal{D}^{\prime}\right) \geqslant d\left(\mathcal{D}, w_{m} x_{0}\right)+d\left(w_{m} x_{0}, w_{n} \mathcal{D}^{\prime}\right)-2\left(y \cdot y^{\prime}\right)_{w_{m} x_{0}}-B,
$$

where $B$ is a constant which only depends on $\delta$ and the quasiconvexity constant $Q$. By Proposition 7 line (7), the difference between the Gromov products $\left(y \cdot y^{\prime}\right)_{w_{m} x_{0}}$ and $\left(x_{0} \cdot w_{n} x_{0}\right)_{w_{m} x_{0}}$ is bounded, as $d\left(w_{n} x_{0}, w_{n} \mathcal{D}^{\prime}\right)=d\left(x_{0}, \mathcal{D}^{\prime}\right)$, and we have chosen a basepoint $x_{0}$ which lies in both $\mathcal{D}$ and $\mathcal{D}^{\prime}$. This implies

$$
d\left(\mathcal{D}, w_{n} \mathcal{D}^{\prime}\right) \geqslant d\left(\mathcal{D}, w_{m} x_{0}\right)+d\left(w_{m} x_{0}, w_{n} \mathcal{D}^{\prime}\right)-2\left(x_{0} \cdot w_{n} x_{0}\right)_{w_{m} x_{0}}-2 C-B,
$$


where $C$ is a number which only depends on $\delta$ and $Q$. The three events whose probabilities are estimated in lines (23), (24) and (26) need not be independent, but the probability that at least one of them occurs is at most the sum of the probabilities that each occurs. Therefore

$$
\mathbb{P}\left(d\left(\mathcal{D}, w_{n} \mathcal{D}^{\prime}\right) \leqslant L_{1} n / 4\right) \leqslant K c^{n / 2}+\check{K} \check{c}^{n / 2+1}+K_{1} c_{1}^{L_{1} n / 4-C-B / 2},
$$

for all $n \geqslant N$, which decays exponentially in $n$, as required.

\section{REFERENCES}

[AM90] Selman Akbulut and John D. McCarthy, Casson's invariant for oriented homology 3spheres, Mathematical Notes, vol. 36, Princeton University Press, Princeton, NJ, 1990.

[BHM11] Sébastien Blachère, Peter Haïssinsky, and Pierre Mathieu, Harmonic measures versus quasiconformal measures for hyperbolic groups, Ann. Sci. Éc. Norm. Supér. (4) 44 (2011), no. 4, 683-721.

[BH99] Martin R. Bridson and André Haefliger, Metric spaces of non-positive curvature, Grundlehren der Mathematischen Wissenschaften [Fundamental Principles of Mathematical Sciences], vol. 319, Springer-Verlag, Berlin, 1999.

[BS09] J. Brock and J. Souto, Volume and distances in the pants complex (2009).

[Deh38] M. Dehn, Die Gruppe der Abbildungsklassen, Acta Math. 69 (1938), no. 1, 135-206.

[DT06a] Nathan M. Dunfield and William P. Thurston, Finite covers of random 3-manifolds, Invent. Math. 166 (2006), no. 3, 457-521.

[DT06b] Nathan M. Dunfield and Dylan P. Thurston, A random tunnel number one 3-manifold does not fiber over the circle, Geom. Topol. 10 (2006), 2431-2499.

[DW11] Nathan M. Dunfield and Helen Wong, Quantum invariants of random 3-manifolds, Algebr. Geom. Topol. 11 (2011), no. 4, 2191-2205.

[Erd59] P. Erdős, Graph theory and probability, Canad. J. Math. 11 (1959), 34-38.

[FM12] Benson Farb and Dan Margalit, A primer on mapping class groups, Princeton Mathematical Series, vol. 49, Princeton University Press, Princeton, NJ, 2012.

[GdlH90] É. Ghys and P. de la Harpe (eds.), Sur les groupes hyperboliques d'après Mikhael Gromov, Progress in Mathematics, vol. 83, Birkhäuser Boston Inc., Boston, MA, 1990 (French). Papers from the Swiss Seminar on Hyperbolic Groups held in Bern, 1988.

[Ham06] Ursula Hamenstädt, Train tracks and the Gromov boundary of the complex of curves, Spaces of Kleinian groups, London Math. Soc. Lecture Note Ser., vol. 329, Cambridge Univ. Press, Cambridge, 2006, pp. 187-207.

[Hem76] John Hempel, 3-Manifolds, Princeton University Press, Princeton, N. J., 1976. Ann. of Math. Studies, No. 86.

[Hem01] John Hempel, 3-manifolds as viewed from the curve complex, Topology 40 (2001), no. 3, 631-657.

[Iva92] Nikolai V. Ivanov, Subgroups of Teichmüller modular groups, Translations of Mathematical Monographs, vol. 115, American Mathematical Society, Providence, RI, 1992. Translated from the Russian by E. J. F. Primrose and revised by the author.

[Joh83] Dennis Johnson, The structure of the Torelli group. I. A finite set of generators for $\mathcal{I}$, Ann. of Math. (2) 118 (1983), no. 3, 423-442.

[Ker90] Steven P. Kerckhoff, The measure of the limit set of the handlebody group, Topology 29 (1990), no. 1, 27-40.

[Kla] E. Klarreich, The boundary at infinity of the curve complex and the relative Teichmüller space. 
[Kob88a] Tsuyoshi Kobayashi, Heights of simple loops and pseudo-Anosov homeomorphisms, Braids (Santa Cruz, CA, 1986), Contemp. Math., vol. 78, Amer. Math. Soc., Providence, RI, 1988, pp. 327-338.

[Kob88b] Tsuyoshi Kobayashi, Casson-Gordon's rectangle condition of Heegaard diagrams and incompressible tori in 3-manifolds, Osaka J. Math. 25 (1988), no. 3, 553-573.

[Kow08] E. Kowalski, The large sieve and its applications, Cambridge Tracts in Mathematics, vol. 175, Cambridge University Press, Cambridge, 2008. Arithmetic geometry, random walks and discrete groups.

[LL10] Gregory F. Lawler and Vlada Limic, Random walk: a modern introduction, Cambridge Studies in Advanced Mathematics, vol. 123, Cambridge University Press, Cambridge, 2010.

[Lic64] W. B. R. Lickorish, A finite set of generators for the homeotopy group of a 2-manifold, Proc. Cambridge Philos. Soc. 60 (1964), 769-778.

[Lin92] Torgny Lindvall, Lectures on the coupling method, Wiley Series in Probability and Mathematical Statistics: Probability and Mathematical Statistics, John Wiley \& Sons Inc., New York, 1992. A Wiley-Interscience Publication.

[Lov68] L. Lovász, On chromatic number of finite set-systems, Acta Math. Acad. Sci. Hungar. 19 (1968), 59-67.

[LPS88] A. Lubotzky, R. Phillips, and P. Sarnak, Ramanujan graphs, Combinatorica 8 (1988), no. 3, 261-277.

[Mah10] Joseph Maher, Random Heegaard splittings, J. Topol. 3 (2010), no. 4, 997-1025.

[Mah11] Joseph Maher, Random walks on the mapping class group, Duke Math. J. 156 (2011), no. 3, 429-468.

[Mah12] Joseph Maher, Exponential decay in the mapping class group, J. Lond. Math. Soc. (2) 86 (2012), no. 2, 366-386.

[MM86] Darryl McCullough and Andy Miller, The genus 2 Torelli group is not finitely generated, Topology Appl. 22 (1986), no. 1, 43-49.

[MT07] John Morgan and Gang Tian, Ricci flow and the Poincaré conjecture, Clay Mathematics Monographs, vol. 3, American Mathematical Society, Providence, RI, 2007.

[Mor97] Shigeyuki Morita, Casson invariant, signature defect of framed manifolds and the secondary characteristic classes of surface bundles, J. Differential Geom. 47 (1997), no. 3, 560-599.

[PH92] R. C. Penner and J. L. Harer, Combinatorics of train tracks, Annals of Mathematics Studies, vol. 125, Princeton University Press, Princeton, NJ, 1992.

[Riv08] Igor Rivin, Walks on groups, counting reducible matrices, polynomials, and surface and free group automorphisms, Duke Math. J. 142 (2008), no. 2, 353-379.

[Sav02] Nikolai Saveliev, Invariants for homology 3-spheres, Encyclopaedia of Mathematical Sciences, vol. 140, Springer-Verlag, Berlin, 2002. Low-Dimensional Topology, I.

[ST06] Martin Scharlemann and Maggy Tomova, Alternate Heegaard genus bounds distance, Geom. Topol. 10 (2006), 593-617 (electronic).

[Woe00] Wolfgang Woess, Random walks on infinite graphs and groups, Cambridge Tracts in Mathematics, vol. 138, Cambridge University Press, Cambridge, 2000.

Alexander Lubotzky

Hebrew University

alex.lubotzky@mail.huji.ac.il 
Joseph Maher

CUNY College of Staten Island and CUNY Graduate Center

joseph.maher@csi.cuny.edu

Conan $\mathrm{Wu}$

Princeton University

conan777@gmail.com 